\title{
MRI Changes and Complement Activation During Epileptogenesis in a Mouse Model of Temporal Lobe Epilepsy
}

Irina Kharatishvili", Zuyao Y Shan*, David T She*, Samuel Foong ${ }^{*}$, Nyoman D Kurniawan*, David C Reutens ${ }^{*}, \#$

* Centre for Advanced Imaging, The University of Queensland, St Lucia, QLD 4072, Australia

\# The Australian Mouse Brain Mapping Consortium, The University of Queensland, St Lucia, QLD 4072, Australia

\author{
Corresponding author: \\ Irina Kharatishvili, \\ E-mail: $\underline{\text { i.kharatishvili@uq.edu.au }}$ \\ Tel: +61 (0)7 33660363 \\ Fax: +61 (0)7 33460330
}

The total number of words of the manuscript, including entire text from title page to figure legends: 8970

The number of words of the abstract: 192

The number of figures: 9

The number of tables: 2 


\section{${ }^{1}$ Abstract}

The complex pathogenesis of temporal lobe epilepsy (TLE) includes neuronal and glial pathology, synaptic reorganization and an immune response. However, the spatio-temporal pattern of structural changes in the brain that provide a substrate for seizure generation and modulate the seizure phenotype is yet to be completely elucidated. We used quantitative MRI to study structural changes triggered by status epilepticus (SE) and their association with epileptogenesis and with activation of complement component 3 (C3). SE was induced by injection of pilocarpine in CD1 mice. Quantitative diffusion-weighted imaging and T2 relaxometry was performed using a 16.4 Tesla MRI scanner at 3 hours and 1, 2, 7, 14, 28, 35 and 49 days post-SE. Following longitudinal MRI examinations, spontaneous recurrent seizures and interictal spikes were quantified using continuous video-EEG monitoring. Immunohistochemical analysis of $\mathrm{C} 3$ expression was performed at 48 hours, 7 days and 4 months post-SE. Animals which developed spontaneous recurrent seizures following SE had specific transient and long-term structural changes within the hippocampus, amygdala, parahippocampal and piriform cortex that were not observed in nonepileptic mice. Chronic $\mathrm{C} 3$ upregulation was found in the hippocampus of epileptic animals within the areas of the persistent MRI changes.

Keywords: epileptogenesis, spontaneous recurrent seizures, temporal lobe epilepsy, magnetic resonance imaging, complement, hippocampus, parahippocampal cortex, epilepsy animal models.

${ }^{1}$ Abbreviations: TLE, temporal lobe epilepsy; SE, status epilepticus; C3, complement component 3; MRI, magnetic resonance imaging; SRS, spontaneous recurrent seizures; DWI, diffusionweighted imaging; NS, no seizures; $\mathrm{CN}$, control; ADC, apparent diffusion coefficient; $\mathrm{T} 2$, apparent transverse relaxation time; $\mathrm{CA} 1$, Cornu Ammonis 1 region of the hippocampus proper; gcl, granule cell layer; $\mathrm{H}$, hilus of the dentate gyrus. 


\section{Introduction}

Temporal lobe epilepsy (TLE), the most common form of refractory focal epilepsy in adults, is associated with widespread structural changes in neuronal networks of the hippocampus and medial temporal lobe (Engel et al. 2008; Bonilha et al. 2012). Mesial temporal sclerosis with neuronal loss and gliosis in the hippocampus, is the most common pathology associated with TLE and can often be detected on visual inspection of clinical MRI scans. However, more detailed morphometric studies have consistently demonstrated changes in extra-hippocampal and extratemporal limbic structures in patients with refractory TLE (Keller et al. 2002; Bonilha et al. 2003; Bernasconi et al. 2004; McDonald et al. 2008).

In most affected patients, TLE is thought to be initiated by insults such as febrile convulsions, status epilepticus, encephalitis or traumatic brain injury (French et al. 1993; Mathern et al. 1995; VanLandingham et al. 1998), following which patients enter a latent period. During this latent period, structural and physiological changes in the brain render it capable of generating recurrent spontaneous seizures, a process referred to as epileptogenesis (Pitkanen 2010). Structural changes that contribute to epileptogenesis are the focus of active investigation and are thought to include neurodegeneration, neurogenesis, gliosis, axonal damage and/or sprouting, dendritic plasticity, changes in the extracellular matrix and angiogenesis (Herman 2006; Pitkanen and Lukasiuk 2011). Emerging evidence from both experimental and clinical gene expression studies strongly indicates activation of innate immunity and particularly of the complement system in association with TLE (Jamali et al. 2006, 2010; Pereira et al. 2005; Aronica et al. 2007; van Gassen et al., 2008). It has been hypothesised that activation of the complement cascade contributes to the development and perpetuation of increased seizure susceptibility in the epileptic focus.

Epileptogenesis is a dynamic process and its relationship with evolving structural changes in different brain regions after the initial injury is not well understood. A better understanding of this relationship is needed to develop novel targeted therapies but prospective population-based studies in humans are scarce and limited by small patient cohorts and short follow-up periods. Animal models that mimic the behavioral and neurophysiological features of human TLE provide an excellent alternative to study the process of epileptogenesis. Chronic epilepsy models, particularly models based on an inciting event of limbic status epilepticus (SE) have been long regarded as highly isomorphic with the human disease since they reproduce most of the key clinical and neuropathologic characteristics of TLE. An initial insult (SE) is followed by a latent period of variable duration that culminates in the development of spontaneous recurrent seizures (SRS). Histological changes such as neuronal death, neurogenesis, synaptic reorganization, reactive gliosis and inflammation are observed in a number of cortical and subcortical brain regions and continue long after the termination of the initial insult (White 2002; Buckmaster 2004; Sharma et al. 2007).

In this study, we examined the time course and pattern of structural changes and complement C3 activation following the precipitating injury in an experimental model of TLE in mice. We studied the relationship between these changes and epileptogenesis by evaluating their correlation with 
electrophysiological characteristics of the epileptic syndrome. Administration of the chemoconvulsant muscarinic agonist pilocarpine induced SE followed by SRS in mice (Cavalheiro et al. 1996; Shibley and Smith 2002). As in the clinical scenario in which not all individuals that incur an initial insult develop spontaneous seizures, in this model at lower doses of pilocarpine, only $30-40 \%$ of animals become epileptic (Cavalheiro et al. 1996; Shibley and Smith 2002; Borges et al. 2003; Curia et al. 2008). This feature of the model permitted us to compare subjects with different outcomes (epilepsy vs. no epilepsy) following the same type of brain injury.

We used high-resolution quantitative diffusion-weighted imaging (DWI) and T2 relaxation measurements to assess structural alterations at different time-points after pilocarpine administration. These parameters were chosen because previous studies showed that T2 relaxometry detects hippocampal sclerosis with high sensitivity in TLE patients (Jackson et al. 1993; Kuzniecky et al. 1997; Briellmann et al. 2002; Pell et al. 2004) and that DWI is a sensitive marker of acute brain injury in both humans and experimental TLE models (Nakasu et al. 1995; Wang et al. 1996; Wall et al. 2000; Fabene et al. 2006). Radiologically, hippocampal sclerosis is indicated by the combination of increased hippocampal T2-weighted signal and decreased hippocampal volume (Jackson et al. 1990), so we performed MRI-based volumetric analysis of the hippocampus. We also studied the expression of complement $\mathrm{C} 3$, the key molecule in the complement system that ultimately drives its effector functions, during the acute, sub-acute and chronic post-injury phases. The relationship between imaging, histological findings and electrophysiological changes was evaluated.

\section{Materials and methods}

\section{Animals}

The present study was approved by The University of Queensland's Institutional Animal Ethics Committee (UQ AEC Certificate CAI/359/10/NHMRC). Seventy seven male, outbred CD1 mice weighing $34 \pm 3.2 \mathrm{~g}$ and aged 7-8 weeks were included in this study. Animals were kept under controlled laboratory conditions (12 hours light/12 hours dark cycle with lights on at 07:00 a.m., temperature $22 \pm 1^{\circ} \mathrm{C}$, air humidity 50-60\%), and had ad libitum access to food and water. The mice were housed individually and given 72 hours to acclimate before the experiment.

\section{Induction of epilepsy}

The procedure for creating the model has been described in detail by Borges et al. (2003). Briefly, mice were injected with methylscopolamine $(1 \mathrm{mg} / \mathrm{kg}$ each i.p. in sterile $0.9 \% \mathrm{NaCl}$, SigmaAldrich, St Louis, MO, USA) 30 min prior to administration of pilocarpine to minimize peripheral cholinergic effects. Experimental animals were then injected subcutaneously with a single dose of pilocarpine (330 mg/kg, in sterile 0.9\% NaCl, Sigma-Aldrich, St Louis, MO, USA). This dose of pilocarpine was chosen because it caused SE in only a fraction of CD1 mice (37\%) in a pilot 
study. Development of SE was visually observed for 90 min after pilocarpine injection and the onset and severity of behavioural manifestations were recorded. Seizures were scored according to the Racine scale (Racine 1972) with slight modifications (Borges et al. 2003): normal activity (stage 0); rigid posture and/or immobility (stage 1); stiffened, extended, arched (Straub) tail (stage 2 ); unilateral forelimb clonus or head bobbing (stage 3); whole body continuous clonic seizures with retained posture (stage 3.5); bilateral forelimb clonus and rearing (stage 4); rearing and falling (stage 5); and tonic-clonic seizures with loss of postural control or jumping (stage 6). Stage 3.5-6 seizures were considered generalized (Shibley and Smith 2002).

SE was defined as at least $30 \mathrm{~min}$ of continuous seizure activity in which seizures were at least stage 3.5 in severity with one or more stage 5 or 6 seizures, or a minimum of 3 stage 4-6 seizures. After 90 min of behavioral SE, seizures were terminated by injection of $25 \mathrm{mg} / \mathrm{kg}$ pentobarbital (i.p.). Mice showing no behavioral seizure activity or less than 3 generalized seizures during 1.5 hours of observation constituted the 'no SE' (NS) group and received an equal dose of pentobarbital 90 min after pilocarpine injection. After SE, all mice were injected with 0.5-1 ml 5\% glucose solution s.c. Mice were fed moistened high-fat rodent chow and monitored daily until they re-gained their preoperative body weight. Sham controls were 5 age- and weight-matched mice injected with equal doses of methylscopolamine and pentobarbital, but with an equivalent volume of saline instead of pilocarpine. Five mice served as naive controls.

\section{MRI}

After the termination of SE, 40 animals (17 SE, 13 NS mice and 10 controls) were randomized into two groups for MR imaging at different time-points. Group I was imaged at 3 hours and 7, 28 and 49 days and Group II was imaged at 1, 2, 14 and 35 days after pilocarpine injection. The length of the follow-up period was designed to cover the latent phase of epileptogenesis, lasting up to approximately 50 days in this model (Cavalheiro et al. 1996; Shibley and Smith 2002).

MRI scans were acquired on a 16.4 Tesla $89 \mathrm{~mm}$ vertical bore scanner interfaced with an Avance II spectrometer (Bruker Biospin, Karlsruhe, Germany). Prior to scanning, mice were anesthetized with $4 \%$ isoflurane in $2.0 \mathrm{~L} / \mathrm{min} \mathrm{O}_{2}$, and $1 \mathrm{ml}$ of sterile $5 \%$ glucose solution was injected subcutaneously to maintain the physical condition of the mice during scanning. Each mouse was placed in a prone position, in a custom made MRI compatible Micro2.5Animal Handling System body and head restraint (M2M Imaging, Brisbane, Australia), to allow repeatable positioning and to minimize motion artefacts. Inside the scanner, anaesthesia was maintained with $1.5-2.0 \%$ isoflurane in $\mathrm{O}_{2}$ at $1 \mathrm{~L} / \mathrm{min}$. Respiration was monitored with Biotrig (SpinSystems, Brisbane, Australia) and maintained at approximately 60-80 breaths per minute by adjusting the level of isoflurane and oxygen flow. A custom-built, $20 \mathrm{~mm}$ SAW volume head coil was used for both excitation and detection. The field-of-view (FOV) and the coronal position of the slices were adjusted to the axes of Paxinos' mouse brain atlas (Paxinos and Franklin 2001), which are similar to the axes used by the Australian Mouse Brain Consortium atlas.

Quantitative T2 MR images were acquired using a Multi-Slice-Multi-Echo sequence with TR = 2

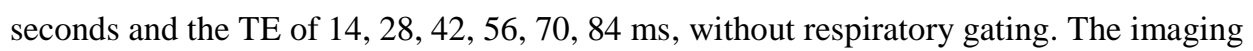


parameters were: $\mathrm{FOV}=1.5 \times 1.5 \mathrm{~cm}$, matrix size $256 \times 256$ to produce a voxel size of $59 \times 59$ micron $\times 1 \mathrm{~mm}$ thickness (9 contiguous coronal slices). The total acquisition time was 8.5 mins. 2D coronal Stejskal-Tanner spin-echo diffusion-weighted images were acquired with respiratory gating using the sequence parameters: $\mathrm{TR}=1.5 \mathrm{~s}, \mathrm{TE}=20 \mathrm{~ms}$, and $\delta / \Delta=2 / 12 \mathrm{~ms}$. Multiple gradient strengths were applied in the slice direction to produce b-values of 0, 400, 800 and $1200 \mathrm{~s} / \mathrm{mm}^{2}$. The same image slice, position and FOV was applied as the T2-map, with matrix size $128 \times 128$ to produce a voxel size of $117 \times 117$ micron $\times 1 \mathrm{~mm}$ thickness ( 9 contiguous coronal slices). The total acquisition time was 17 mins.

\section{Image analysis}

T2 relaxation times were calculated by linear fitting of image intensity at different echo times using the formula $\ln (S)=\ln \left(S_{0}\right)-t / T 2$ in which $S$ is the image intensity, So is the initial intensity and $t$ is the echo time. The apparent diffusion coefficient (ADC) at each voxel was calculated using the formula $\ln (S)=\ln \left(S_{0}\right)-b * A D C$.

Image preprocessing was performed with MIPAV4.4.1 (NIH, Bethesda, USA). The T2 images with echo time of $14 \mathrm{~ms}$ for each animal were used as the anatomic images for each mouse. Other images, including the T2 images with different echo times and DWI data, were registered to the corresponding anatomic images using rigid body registration based on normalized mutual information (NMI). A knowledge-guided active contour method was used to segment out the brain on anatomic images: 1) An averaged anatomic image was generated using two-pass rigid body and affine registrations to the anatomic images of the control animals; 2) the initial brain contour was manually defined on the averaged anatomic images; 3) the brain contour was transferred to the individual mouse images using the matrix found by rigid body registration of the averaged anatomic images to individual anatomic images; 4) the brain contour was actively adjusted to locate the individual brain contour using an energy minimizing B-spline method (Shan et al. 2005). The brain masks found on anatomic images were applied to the T2 and ADC maps. Individual T2 and ADC brain maps were then normalized to the Australian Mouse Brain Mapping Consortium (AMBMC) brain template (Ullmann et al. 2012) using the transformation matrix found by a nonlinear cubic B-spline registration of individual anatomic brain images to the brain template using NMI.

Statistical parametric mapping with SPM8 (Wellcome Trust Centre for Neuroimaging, UK) was used to compare ADC and T2 maps between 3 different groups: control animals $(\mathrm{CN})$, pilocarpine-treated animals with SE (SE), and pilocarpine-treated animals with no SE (NS). The analyses were performed on registered images smoothed with a 1mm FWHM Gaussian kernel. Voxel-based unpaired $t$-tests were used for group comparisons to identify the locations of significant differences in $\mathrm{T} 2$ and ADC at each time-point. The relative T2 and ADC changes at each voxel in the SE mice were also correlated with spike frequency in the randomly selected 96 hour interval. Relative ADC values were calculated using:

$$
\Delta A D C_{i, S E}=\left(A D C_{i, S E}-\overline{A D C_{i, C N}}\right) \times 100 \% / \overline{A D C_{i, C N}}
$$


in which $A D C_{i, S E}$ and $\overline{A D C_{i, C N}}$ are the ADC value of the ith voxel in an individual subject in the SE group and the mean ADC value in the ith voxel in the control group, respectively. The relative T2 map was calculated similarly. For these analyses, a threshold following correction for multiple comparisons was selected for False Discovery Rate (FDR) <0.05 (Genovese et al., 2002).

\section{Relative hippocampal volumes}

Since hippocampal sclerosis (HS) is the most frequent lesion found in TLE, we also performed region of interest (ROI) -based analysis focusing on the hippocampus using MIPAV4.4.1 (NIH, Bethesda, USA). All slices containing the hippocampus (bregma levels $2.80 \mathrm{~mm}$ and $-4.36 \mathrm{~mm}$ ) on MR images for each mouse at each time-point were employed in this analysis. A blinded investigator manually delineated the left and right hippocampi and the whole brain on the anatomic images in native space. The relative hippocampal volumes were calculated as the ratio of the hippocampal volumes to the brain volumes in the respective slices. Statistical analyses were performed with SPSS for Windows (v.19.0) and the threshold for statistical significance was set at $\mathrm{p}<0.05$. Kruskal-Wallis ANOVA was used to test for significance. If significance group differences were found on ANOVA, the Mann-Whitney $U$ test was performed as a post-hoc test to assess differences between two specific groups.

\section{Electrode implantation}

To detect and quantify SRS and to correlate epileptogenicity with MRI changes, we performed continuous video-EEG monitoring on 30 pilocarpine-injected animals and 4 controls for 3 consecutive weeks. Five to 7 weeks after the induction of SE, the animals were anesthetized with a single i.p. injection $(60 \mathrm{mg} / \mathrm{kg})$ of sodium pentobarbital. The head of the mouse was secured in a stereotaxic apparatus (lambda and bregma on the same horizontal level) and the skull periosteum exposed by a midline skin incision. Two stainless steel epidural screw electrodes $(1 \mathrm{~mm}$ diameter, Microbiotech/SE AB, Sweden) were placed over the left and right parietal cortex. Two additional screw electrodes were inserted into the skull over the cerebellum bilaterally and served as ground and indifferent electrodes. All electrode pins were inserted into the plastic pedestal (Plastics One Inc., Roanoke, VA, USA) and the entire assembly was cemented to the calvarium with dental acrylic (Vertex Castapress, Vertex-Dental B.V., Zeist, Netherlands). Five days after surgical procedures, the EEG was recorded in freely moving mice 24 hours/day for 3 consecutive weeks 10 to 11 weeks after SE to detect SRS. Continuous video-EEG monitoring was performed using a Compumedics 32 Channel E-Series and a ProFusion EEG recording system with digital video, connected to an E-series 32 channel amplifier (Compumedics Ltd, Abbotsford, Victoria, Australia), using a sampling rate of $256 \mathrm{~Hz}$. EEG recordings were filtered with a filter with $0.3 \mathrm{~Hz}$ high-pass and $100 \mathrm{~Hz}$ low-pass cut-offs. Mice were placed in plexiglass cages $(30$ x 18 x $21 \mathrm{~cm})$ where they could move freely (one mouse per cage) and connected to the EEG recording system with commutators (Plastics One Inc., VA, USA). The behaviour of the animals was recorded using 
2 CCD video cameras (Panasonic SD5 -S76250) positioned in front of the cages. The system allowed simultaneous video- EEG recording of sixteen animals at a time.

\section{Analysis of video-EEG}

Digital EEG files were analysed manually using proprietary ProFusion EEG software. An electrographic seizure was defined as a high amplitude rhythmic discharge or spike and wave pattern with a clear onset, offset, and temporal evolution in frequency and/or morphology, lasting at least $10 \mathrm{~s}$. Behavioral severity of seizures was assessed on video according to the modified Racine scale (Racine 1972). A spike was defined as a paroxysmal potential with sharp contour and a duration of 20-70 ms, usually followed by a low-voltage slow potential (about $200 \mathrm{~ms}$ duration). The number of spikes was counted manually from a 96 hour period beginning at a randomly chosen starting point in the EEG recording.

\section{Histology}

Mice were deeply anesthetized using an intraperitoneal injection of $100 \mu 1$ of Virbac ${ }^{\circledR}$ Lethabarb $(325 \mathrm{mg} / \mathrm{ml}$ sodium pentobarbitone, Peakhurst, NSW, Australia). The animals were then transcardially perfused through the left ventricle and ascending aorta with ice-cold phosphate buffered saline (PBS, 0.01M, pH 7.4) followed by ice-cold 4\% paraformaldehyde (PFA) in $0.01 \mathrm{M}$ PBS. After post-fixation in 4\% PFA for 24 hours, the brains were stored at $4{ }^{\circ} \mathrm{C}$ in $1 \mathrm{XPBS}$ with $0.05 \%$ sodium azide until being sectioned in coronal plane using a vibratome (VT 1000S, Leica Microsystems GmbH, Wetzlar, Germany, 40 $\mu$ m thickness 1-in-12 series).

\section{Immunohistochemistry}

In the early post-injury phase, serial sections were analysed from representative mice 48 hours $(n=3)$ and 7 days $(n=3)$ after the injection of pilocarpine and SE lasting at least $1 \mathrm{~h}$. The timepoints were chosen as corresponding to the times of critical MRI changes. In the chronic postinjury phase, serial sections were analysed from mice with electrographically confirmed epilepsy $(n=8)$, mice without epilepsy $(n=8)$, and untreated controls $(n=5)$. Free-floating sections were washed for $15 \mathrm{~min}$ in $0.01 \mathrm{M}$ PBS (pH 7.4) followed by a 15 min wash in 50\% ethanol. The sections were then pre-treated in $1 \mathrm{X}$ PBST $(0.15 \%$ Triton X-100 in $1 \mathrm{X}$ PBS, pH 7.4) for 15 mins. Non-specific staining was minimized by incubating sections in $10 \%$ donkey serum (DS) in $1 \mathrm{X}$ PBST ( $\mathrm{pH}$ 7.4) for 2 hours. Sections were then incubated in a solution containing rat monoclonal antibody against mouse native $\mathrm{C} 3$ and its activation products, $\mathrm{C} 3 \mathrm{~b}, \mathrm{iC} 3 \mathrm{~b}, \mathrm{C} 3 \mathrm{~d}$ and $\mathrm{C} 3 \mathrm{dg}(11 \mathrm{H} 9$ dilution: 1:100, Hycult Biotech, Uden, The Netherlands) for 48 hours at $4^{\circ} \mathrm{C}$. For double-labelling of $\mathrm{C} 3$ with markers for astrocytes, neurons or microglia the sections were incubated with the following primary antibodies: mouse polyclonal antibody against glial fibrillary acidic protein (GFAP, dilution: 1:1000, Millipore, Billerica, MA, USA), mouse monoclonal antibody against neuronal nuclei (NeuN, dilution 1:1000, Millipore), or rabbit polyclonal antibody against ionized 
calcium binding adapter molecule 1 (Iba1, dilution: 1:1000, Wako Pure Chemical Industries, Ltd., Osaka, Japan) for $2 \mathrm{~h}$ at room temperature with gentle agitation. The primary antibodies were diluted in $1 \mathrm{X}$ PBST and $2 \%$ DS.

After washes with 1X PBST, sections were incubated for 2 hours at room temperature in either Alexa Fluor 488 Goat Anti-Mouse IgG (dilution: 1:1000, Life Technologies Corp, Grand Island, NY, USA) or Alexa Fluor 488 Goat Anti-Rabbit IgG (dilution: 1:1000, Life Technologies Corp) diluted in IX PBST to visualise astrocytes, neurons or microglia. After being washed, sections were then incubated for 2 hours at room temperature in Alexa Fluor 594 Donkey Anti-Rat IgG (dilution: 1:1000, Life Technologies Corp) to visualise complement C3. The nuclei were counterstained with DAPI (dilution: 1:1000, Life Technologies Corp). Sections were then mounted on charged glass slides (superfrost plus) and coverslipped with Mowiol-488 for further analysis. Sections were examined using a light microscope (Axio Imager 2, Carl Zeiss Microscopy GmbH, Jena, Germany) with an ApoTome attachment (Axio Imager z1 stand). Fluorescence images were captured using a monochrome CCD camera (AxioCam MRm, Zeiss). The ApoTome structuredillumination attachment allowed optical sectioning using the CCD camera. The AxioVision software allowed automatic collection of multi-channel Z-stack images. Images were acquired with $5 x$ and 20x lenses.

\section{Results}

\section{Status epilepticus (SE)}

Data are expressed as mean \pm standard deviation. Behavioral manifestations included immobility followed by body tremor with a latency of $8 \pm 3 \mathrm{~min}$, that later developed in the following stages: mouth and facial automatisms, head nodding, unilateral forelimb clonus, bilateral forelimb clonus with rearing, rearing and falling/loss of postural control. Mean latency to first seizure was $23.5 \pm 3.4 \mathrm{~min}$. Forty two percent of injected mice (28 out of 67 ) experienced and survived behavioral status epilepticus (SE) as defined by continuous behavioral seizure activity consisting of mainly generalized clonic seizures and lasting at least 0.5 hour. Stage 3-5 seizures were typically of 30-90 seconds duration and were separated by periods of continuous stage 1 and 2 seizure activity. The rest of the mice did not show any behavioural manifestations above score 1 or had 1-2 isolated seizures (score 2-3) during the 90 min observation period. Control animals receiving methylscopolamine and saline did not exhibit any behavioural alterations. The acute mortality rate for the pilocarpine-treated mice was $21 \%$, within the reported range (Shibley and Smith 2002; Borges et al. 2003). 


\section{MR Imaging}

\section{$M R$ diffusion and relaxation changes in limbic regions reflect different outcome}

The numerical values and anatomical locations of significant differences in ADC and T2 between the three groups are summarized in Tables 1 and 2, and Figures 1 and 2, respectively. In the NS group (NS vs CN), MRI changes were confined to the hippocampus and amygdala between the first 24 hours and 7 days most likely reflecting pilocarpine-induced effects, as none of these animals had SE or observed SRS. This early increase in ADC and T2 returned to normal by 2 weeks (Fig. 1A, Fig. 2A). This group did not exhibit MRI changes at later stages. In the SE group ( $\mathrm{SE}$ vs $\mathrm{CN}$ ), in addition to the early hippocampal and amygdalar changes also observed in the NS group, later persistent MRI changes were observed in the parahippocampal and piriform cortices (Fig. 1B, Fig. 2B). Given that they were not present in the NS group, these MRI changes are likely to contain a component that is SE-induced. The changes persisted throughout 7 weeks of MRI follow-up. Since all animals in the SE group had experienced SE and developed SRS (see below: Spontaneous recurrent seizures and interictal epileptiform activity), it was not possible to distinguish the changes caused by SE from those specifically related to epileptogenesis. The comparison of SE vs NS (i.e. epilepsy vs no epilepsy) groups, revealed, however, a pattern of spatio-temporal MRI alterations different from that in SE vs CN comparison, with more widespread and severe damage to the amygdala and piriform cortex and more localized changes in the hippocampus and parahippocampal cortex in the group with epilepsy compared to nonepileptic animals (Tables 1 and 2; Fig. 1C and 2C).

\section{Dynamics of $A D C$ changes in relation to epileptogenesis}

Three to 24 hours post-SE, there was no difference in ADC maps between SE and NS groups. This may be an effect of residual exposure to pilocarpine which has direct neurotoxic and inflammatory effects. Animals in both groups received equal doses of pilocarpine which remains present in blood and brain up to 24 hours after injection (Mazzuferi et al., 2012). Forty eight hours post-SE, an increase in ADC in the piriform, ectorhinal, perirhinal, and entorhinal cortices, amygdala, ventromedial thalamic nuclei and the hippocampus was observed in SE animals (Fig.1C, Table 1). A significant difference in ADC between SE and NS animals was present in the amygdala, piriform and parahippocampal cortices for 2 weeks and subsided by 4 weeks (Fig. 1C, Table 1). In contrast, in the hippocampus, the difference between SE and NS groups in ADC persisted throughout the 7 week imaging period, in keeping with ongoing pathological changes in the hippocampus in SE animals destined to develop epilepsy (Fig. 1C, Table 1).

\section{Dynamics of T2 changes in relation to epileptogenesis.}

Three to 24 hours post-SE, there was no difference in T2 maps between SE and NS groups. At 48 hours, T2 increased in the amygdala in SE compared to NS animals (Fig. 2C, Table 2) and 
remained elevated throughout the 7 week period of observation. In the NS group, no T2 changes in the amygdala were observed at any time-point except 48 hours post-SE (Fig. 2A, Table 2). At 48 hours post-SE, T2 increased in the hippocampus in both SE and NS animals when compared to controls (Fig. 2A and 2B, Table 2). The difference in hippocampal T2 between SE and NS groups appeared later, at day 7. By days 14-35, T2 changes had spread to involve the parahippocampal cortex (Fig. 2C, Table 2). Overall, differences in the hippocampus between the two groups were less prominent on $\mathrm{T} 2$ maps than on ADC maps.

Seven to 14 days post-SE, in addition to changes in the amygdala and hippocampus, SE animals showed increased T2 values in the piriform cortex compared to NS group (Fig. 2C, Table 2). T2 remained elevated to the end of the monitoring period, indicating that $\mathrm{T} 2$ may be more sensitive than ADC to damage in the piriform cortex and amygdala (Fig. 2C, Table 2).

\section{Hippocampal volume analysis}

A consistent decrease in relative hippocampal volume was observed in the SE group compared to NS and control (CN) groups. This decrease started 24 hours after the induction of SE and became significant at 7 days (Fig. 3). No significant difference was found in relative hippocampal volumes between NS and $\mathrm{CN}$ groups.

\section{SRS and interictal epileptiform activity}

\section{Occurrence of SRS}

Monitoring was performed in 14 animals with SE, 16 mice with no SE (NS group) and 4 controls for 21 consecutive days. The NS group included 6 mice that had 1-2 isolated seizures after pilocarpine injection and 10 mice with no behavioural seizures after pilocarpine injection. None of the animals that failed to develop SE after pilocarpine injection exhibited any epileptiform changes on EEG during the entire period of monitoring. Video-EEG confirmed the presence of behavioral and electrographic SRS in 11 of the 14 SE mice (79\%). In the remaining 3 SE animals (21\%), continuous interictal spiking was recorded on EEG but no SRS were captured.

\section{Characteristics of spontaneous chronic seizures}

In mice with SRS, the mean seizure frequency was $0.5 \pm 0.3$ seizures per day (range 0.24 to 0.95 seizures per day) and the mean seizure duration was $51 \pm 4.5$ seconds (range 43.4 to 56.9 seconds). In all animals with SRS, electrographic seizures occurred with behavioural manifestations and were secondarily generalized (behavioural stage 4-5) according to the Racine scale (Racine 1972). In 7 mice, seizures appeared in clusters (up to 4 seizures within a 2 hour recording period). Each animal also showed interictal spiking. Mean spike frequency in the 96 hour spike-counting period was $13.1 \pm 11.5$ spikes per hour (range 1.05 - 39.9 spikes/hour). There was a correlation between the number of spikes in a 96 hour interval and the number of seizures within the 3 week 
monitoring period $(r=0.7 ; p<0.05)$. The $3 \mathrm{SE}$ animals in which SRS were not recorded (an\#20, an\#37, an\#66) had the lowest spike frequency (mean of $1.76 \pm 0.25$ spikes/hour compared to $17.4 \pm 11.5$ spikes/hour in those with observed SRS). Since animals without SE in our study did not show any epileptiform abnormalities on EEG, and animals with lower spike frequency did not exhibit SRS within 3 weeks of monitoring, it is reasonable to conclude that the presence and frequency of spiking activity reflected the severity of ongoing epileptogenesis.

\section{Quantitative MRI changes correlate with epileptogenicity}

Epileptogenicity was defined as the presence and severity of epileptic condition (Engel 2011). In light of the strong correlation between number of spikes and number of seizures, we used the number of interictal spikes as a quantitative descriptor of the intensity of the epileptogenic process. Spike frequency in a randomly selected $96 \mathrm{~h}$ interval was correlated with relative T2 and ADC changes. Regression analysis showed that increased ADC values in the ventral hippocampus (at the level of bregma $-3.4 \mathrm{~mm}$ ) 7 days after SE correlated with spike frequency at the chronic stage ( $<<0.001$; Fig. 4). T2 changes or relative hippocampal volumes at any time point did not correlate with spike frequency.

\section{C3 activation}

Figure 5 shows the pattern of C3 immunoreactivity in the hilar regions 48 hours post-SE. Colocalization studies indicated that C3 was induced in reactive astrocytes, but not neurons or microglia, even though prominent microglial activation was present in all 3 animals. Double label immunostaining 1 week (Fig. 6) and 4 months (Fig. 7) post-SE showed a similar pattern of C3 immunoreactivity despite the presence of activated microglia. The hippocampus from $\mathrm{CN}$ animals showed weak C3 immunoreactivity in the hilar region and staining of blood vessels (data not shown). One week post-SE, when ADC increase in the ventral hippocampal region correlated with spike frequency, prominent reactive gliosis was found in the SE group with reactive astrocytes showing double labelling with C3 (Fig. 8B, C and D). In NS animals there was less pronounced gliosis in the hippocampus than in SE mice and C3 expression was restricted to blood vessels (Fig. $8 \mathrm{E}$ and F). Figure 9 illustrates the pattern of $\mathrm{C} 3$ immunoreactivity in the septal hippocampus in animals from each of the three groups 4 months post-SE. C3 was chronically upregulated in the hippocampus corresponding to the areas of significant MRI changes at 49 days post-SE (Fig. 8A). In the $\mathrm{CN}$ hippocampus ( $\mathrm{n}=5), \mathrm{C} 3$ immunoreactivity was barely detectable (Fig. 8B). The pattern of C3 expression in NS animals $(n=10)$ was similar to controls (Fig. 8C1-C3). In SE animals $(n=14), C 3$ was highly expressed by astrocytes in the hippocampus, particularly in the subgranular zone of the dentate gyrus (Fig. 8D1-D3). In the $3 \mathrm{SE}$ mice that had interictal spiking on EEG but no spontaneous seizures detected during 3 week interval, the pattern of C3 expression was similar to mice with SRS. 


\section{Discussion}

To our knowledge, this is the first study in a mouse TLE model to correlate MRI alterations with epilepsy outcome. MRI changes reflected different results in relation to epilepsy development. We identified ADC increase in the amygdala, piriform and parahippocampal cortices and the septal hippocampus at 48 hours as a potential early biomarker for epileptogenesis and increased ADC in the hippocampus at 7 days as a potential biomarker for the severity of the epileptic condition. C3 activation was found in the sub-acute and chronic stages of epileptogenesis in the hippocampus of SE animals i.e. those destined to develop epilepsy.

\section{MRI changes that precede the onset of epilepsy and correlate with epileptogenicity}

Traditionally, acquired epileptogenesis has been viewed as a finite process spanning a period of months or years between an initial precipitating injury and the onset of spontaneous recurrent seizures (Bragin et al. 2000; Walker et al. 2002; Chang and Lowenstein 2003; Rakhade and Jensen 20099; Giblin and Blumenfeld 2010). More recently, it has been suggested that epileptogenesis also relates to clinical progression in terms of changes in seizure severity and frequency and the development of comorbidities and refractoriness to medication (Pitkanen 2010; Dudek and Staley 2011). An alternate view posits distinct processes: epileptogenesis - the creation of mechanisms necessary for spontaneous epileptiform activity to exist, and epileptic maturation - the processes that mediate evolution in the clinical phenotype of the disorder (Sloviter and Bumanglag 2012). Our data speak to this debate by identifying a consistent link between MRI changes and the development of epilepsy early in the silent period, prior to seizure onset; these changes occurred contemporaneously in a network of limbic structures and reflected epileptogenesis. A different set of MRI changes related to the severity of the epileptic state.

It has been established previously that the latent period in pilocarpine model is highly variable and strain-dependent (Cavalheiro et al. 1996; Shibley and Smith 2002; Chen et al. 2005; Winawer et al. 2007; Mueller et al. 2009). The seizure free latent period in CD1 mice lasts between 4 and 54 days, with a mean of 19 \pm 8.9 days (Shibley and Smith 2002; Curia et al. 2008). In our study, the exact duration of the latent period in individual mice is unknown since concurrent EEG and MRI examinations were unfeasible due to signal drop out and image distortion from magnetic susceptibility effects of EEG electrodes at ultra-high field strength. However, on the basis of previous studies and our own behavioural observations, it is reasonable to assume that at 48 hours post-SE the vast majority, if not all, of the experimental animals were still in the latent phase. At this time-point, we observed differences in $\mathrm{ADC}$ and $\mathrm{T} 2$ between the groups of mice with different seizure outcome. 


\section{Hippocampus}

At 48 hours post-SE, animals destined to develop epilepsy had increased ADC values in the dorsal hippocampus in comparison with non-epileptic animals. Multiple clinical and experimental studies have demonstrated hippocampal involvement in TLE (for review, see McNamara 1994; Pitkanen and Sutula 2002; Sharma et al. 2007). The alterations within specific hippocampal circuits that lead to spontaneous seizures are not completely understood but a key structure is likely to be dentate gyrus, the primary gateway regulating cortical input to the hippocampus (Wuarin and Dudek 2001). During the latent period, the dentate gyrus undergoes a number of histological changes including loss of hilar interneurons, appearance of newly formed ectopic granule cells, and sprouting of mossy fibers. These changes are likely to relate to remodeling of dentatehippocampal circuits during epileptogenesis (Dudek and Sutula 2007; Sharma et al. 2007). However, data from the rat pilocarpine model suggest that the dentate gyrus has comparable electrophysiological responses in both epileptic and control rats (Ang et al. 2006). Alternative cortical pathways innervating the hippocampus may also contribute to seizure initiation (Avoli et al. 2002; Biagini et al. 2005; Bartolomei et al. 2005; Ang et al. 2006).

\section{Parahippocampal structures}

In the early post-SE period, increased ADC in the piriform and parahippocampal cortices and elevated ADC and T2 in amygdala occurred only in a group of mice that later developed epilepsy. Our data are in accordance with a recent MRI study in an electrical stimulation (SE) model in which only the rats with acute parahippocampal damage as evidenced by $\mathrm{T} 2$, diffusivity and anisotropy changes developed spontaneous seizures (Parekh et al. 2010). The importance of parahippocampal structures in the initiation, propagation and manifestation of limbic seizures has been long acknowledged in both experimental and human TLE (Bernasconi et al. 1999; Schwarcz et al. 2002; Pereira et al. 2005; McIntyre and Gilby 2006, 2008). The piriform cortex contains a critical, functionally defined epileptogenic trigger zone, the "Area Tempestas", from which bilateral limbic motor seizures can be evoked (Halonen et al. 1994; Loscher and Ebert 1996; Demir et al. 2000, 2001). The piriform cortex may also function as an amplifier region, increasing and propagating epileptic seizure activity induced in other limbic regions (reviewed in Loscher and Ebert 1996). The perirhinal cortex has major reciprocal projections with piriform, entorhinal and insular cortices (Agster and Burwell 2009), and with basal and lateral amygdalar nuclei (Pikkarainen and Pitkanen 2001). Several lines of evidence point to an important role for the perirhinal cortex in the initiation and spread of seizures. The volume of the perirhinal cortex ipsilateral to the seizure focus is significantly reduced in patients with TLE (Bernasconi et al. 2000, 2003; Keller et al. 2004; Meade et al. 2008). The perirhinal cortex is the most easily kindled forebrain structure (McIntyre et al. 1993; Kelly and McIntyre 1996), and is more likely to generate electrographic seizures in vitro than adjacent limbic structures (De Guzman et al. 2004). A primary role for the perirhinal cortex in seizure generalization has also been reported (Kelly and McIntyre 1996; McIntyre and Kelly 2000). Recent electrophysiological assessment of the perirhinal cortex in pilocarpine SE model showed the presence of spontaneous epileptiform activity in brain slices 
obtained from chronically epileptic rats, but not in those without epilepsy (Benini et al. 2011).The amygdala may also be a site of seizure generation (for review, see Aroniadou-Anderjaska et al. 2008) and in some patients with TLE amygdalectomy alone is sufficient to eliminate seizures (Feindel and Rasmussen 1991; Jooma et al. 1995; Wieser 2000). The basolateral nucleus of the amygdala (BLA) is thought to play a key role in the initiation and spread of seizures (White and Price 1993a, b; Tuunanen et al. 1996, 1997) and is commonly a site of severe neuronal loss and gliosis in TLE patients. Pitkanen et al. (1998) suggested that the preferential loss of inhibitory neurons in amygdalar nuclei facilitates seizure propagation to other brain regions.

Our findings suggest that the substrate for limbic seizure generation involves a widely distributed neural network in keeping with previous observations of multifocal seizure onset in different but highly interconnected limbic structures (Spencer and Spencer 1994; Bertram 1997, 1998) and multifocal anatomical changes in TLE (Bertram and Cornett 1994; Wuarin and Dudek 1996; Natsume et al. 2003; Bertram 2009).

\section{MRI changes that relate to epilepsy severity}

Seven days post-SE, increased ADC in the ventral hippocampus correlated with chronic spike frequency. We postulate that these MRI changes reflect epileptic maturation and the evolution in the clinical phenotype rather than epileptogenesis per se. We used frequency of interictal spikes as an index of the severity of epileptic condition. Spike frequency was strongly correlated with number of SRS, animals without SE did not show any interictal spikes and animals with lower spike frequency did not develop SRS during the 3 week recording period. Several studies have demonstrated a relationship between the frequency of interictal spikes and the frequency of seizures, secondary generalisation and status epilepticus in patients with TLE (Rosati et al. 2003; Clemens et al. 2005; Janszky et al. 2005; Krendl et al. 2008). Interictal spikes may contribute to the development and maintenance of the epileptic state by driving activity-dependent signalling and gene expression (Rakhade et al., 2007).

To date, few other experimental studies have investigated the ability of early MRI changes to predict epileptogenesis or severity of subsequent epilepsy. In a rat lithium-pilocarpine model, a subgroup with increased T2 signal in the piriform and enthorhinal cortices at 24 hours after SE became epileptic, but those without changes in T2 signal did not develop spontaneous seizures (Roch et al. 2002). Early decreases in hippocampal diffusivity correlated with increased seizure susceptibility in a model of post-traumatic epilepsy (Kharatishvili et al. 2007). In the selfsustaining SE rat model, alterations in diffusivity and fractional anisotropy in the amygdala and piriform cortex correlated with the development of spontaneous seizures (Parekh et al. 2010). In contrast to previous imaging studies that have been ROI-based, we used voxel-based statistical parametric mapping to map structural changes longitudinally. The whole brain voxel-based approach has several advantages over ROI-based methods. It does not impose an a priori restriction on the anatomical structures to be interrogated. It is more sensitive to highly localised changes within structures; these may be averaged out with a ROI-based approach. Finally, the ROI-based method is susceptible to intra- and inter-rater variability in the delineation of 
anatomical boundaries. Furthermore, our study is the first one to use long-term video-EEG monitoring for reliable detection and quantification of spontaneous epileptiform activity and subsequent correlation with MRI results.

\section{Complement activation is linked to epilepsy outcome}

Our data confirm and expand on previous observations of a complex and sustained inflammatory reaction that contributes to or modulates epileptogenesis following brain injury (Vezzani and Granata 2005; Marchi et al. 2009, 2011; Vezzani et al. 2008; Vezzani et al. 2011a, b, c).

Activation of the complement system is an important component of the inflammatory response, leading to activation of microglia, secretion of proinflammatory cytokines, recruitment of macrophages, activation of phagocytosis and increased vascular permeability (Lucas et al. 2006). C3 plays a central role in the activation of the complement system and is the point of convergence of classical, alternative and lectin pathways of complement activation. Complement activation is increasingly recognized as playing a key role in the development of TLE. Prominent activation of the classical complement pathway occurs in the hippocampus and entorhinal cortex following SE in animal models (Aronica et al. 2007), and C3 deficient mutant mice are less prone to develop epilepsy and have less severe pathological changes following brain infection (Libbey et al. 2010). Glial and neuronal expression of C3 is observed in regions with neuronal loss in hippocampi from patients with TLE (Aronica et al. 2007) and microarray studies detect C3 upregulation in the enthorhinal cortex (Jamali et al. 2006). A functional polymorphism in the promoter of the C3 gene may also confer susceptibility to TLE following febrile seizures (Jamali et al. 2010). Complement activation regulates cytokine synthesis by monocytes, and in turn, cytokines induce $\mathrm{C} 3$ expression in astrocytes. Thus, reactive astrocytes, which were present within the hippocampus in our study, may represent an additional source of complement components contributing to a chronic, selfpropagating immune response.

We observed persistent and prominent C3 activation in the hippocampus only in SE animals, which, on the basis of observations on others in the cohort, were destined to develop epilepsy. Moreover, we demonstrated the presence of activated C3 in the areas of significant MRI changes correlating with the development of epilepsy. The observation of a similar pattern of complement activation in animals with spontaneous seizures and those with only interictal spikes suggests that C3 activation plays a role in epileptogenesis but it may also be important in epileptic maturation because it was observed in areas where MRI changes correlated with the severity of epilepsy. Further studies of complement activation during both epileptogenesis and epileptic maturation are required to clearly delineate these roles and may help to identify novel therapeutic strategies.

\section{Methodological Issues}

MRI changes reflect contributions from a number of processes that differ at each time point. These include direct neurotoxic and inflammatory effects of systemic pilocarpine injection, structural 
damage secondary to SE and structural changes directly related to epileptogenesis and epilepsy maturation. In previous studies of a pilocarpine model in rats, MRI changes and their regional occurrence depended on the severity of acute seizures (Fabene et al. 2003; Nehlig 2011). In our study, it is possible that in the acute stage (3-24 hours post-SE) inter-animal variability in seizure number and characteristics contributed to variability in the levels of cell loss and cytotoxic edema, affecting the significance of diffusion results. However, the T2 measurements which are less sensitive to this problem also did not reveal any differences between SE and NS animals. To shorten scan acquisition times, diffusion data were acquired with gradients applied in the slice direction only. Thus the maximum sensitivity to changes in diffusion is in one particular orientation and the least sensitivity is orthogonal to this plane. It is possible that diffusion measurements with gradients in other directions may demonstrate additional abnormalities. Most histologic analysis was undertaken at a chronic stage, 12 -16 weeks post-SE, to allow electrographic quantification of the seizure phenotype. Only a small number of representative animals were sacrificed at the two acute time-points that proved significant in the MRI analysis. Further studies with larger cohort and co-registration of MRI with histology will allow a better understanding of the temporal progression and correlation of MRI changes with underlying pathological changes.

\section{Conclusion}

In conclusion, this longitudinal MRI study in a TLE mouse model is the first to relate the early and sub-acute changes in ADC and T2 with long-term epilepsy outcome. Early MRI changes may help to predict the likely development of epilepsy and thereby contribute to the development of diagnostic and preventive strategies. Our observations on complement activation, which occurred exclusively in animals with epilepsy and was present through all stages of epileptogenesis and epileptic maturation, raise questions about the role of innate immunity both in the initiation and in the perpetuation of seizures.

\section{Acknowledgments}

We would like to thank the National Imaging Facility (NIF) and the Queensland NMR Network (QNN) for access to the 16.4 T scanner and technical support. We thank Australian Mouse Brain Mapping Consortium for providing an atlas for image registration. We express our gratitude to Dr Karin Borges for advice in establishing a mouse pilocarpine model, and to Ms Jane Ellis and Mr. Luke Hammond from Queensland Brain Institute for technical advice and assistance. This project was funded by the National Health and Medical Research Council (NHMRC) of Australia (to D.C.R) and the University of Queensland New Staff Research Start Up Grant (to I.K.). 


\section{Figure legends}

\section{Figure 1}

Progression of ADC changes during epileptogenesis and epileptic maturation in a mouse pilocarpine TLE model. Voxel-based comparison of ADC maps generated for 3 groups of animals: controls $(\mathrm{n}=10)$, mice that experienced status epilepticus after pilocarpine injection (SE group, $\mathrm{n}=17$ ), and mice without SE (NS group, $\mathrm{n}=13$ ). Data are presented in chronological order from 3 hours to 49 days after the initial insult. Only the slices showing group differences at any one timepoint are shown. (A) Sequence of ADC changes in NS group compared to control animals. (B) Spatio-temporal evolution of differences in ADC between SE and control groups. (C) Sequence of ADC changes in SE group compared to NS animals. The color scale bars on the left represent the $\mathrm{T}$ value, which can be negative (colour-coded from blue to yellow) or positive colour-coded from green to red). The $\mathrm{T}$ values were calculated as ratios of differences between the two groups to the standard deviation of the two groups.

\section{Figure 2}

Progression of $\mathrm{T} 2$ changes during epileptogenesis and epileptic maturation in a mouse pilocarpine TLE model. Voxel-based comparison of T2 maps generated for 3 groups of animals: controls, mice that experienced status epilepticus (SE) after pilocarpine injection (SE group) and mice without SE (NS group). Data are presented in chronological order from 3 hours to 49 days after the initial insult. Only the slices showing group differences at any one time-point are shown. (A) Sequence of T2 changes in NS group compared to control animals. (B) Spatio-temporal evolution of differences in T2 between SE and control groups. (C) Sequence of T2 changes in SE group compared to NS animals. Scale: the significance level of T values (ratios of differences between the two groups to the standard deviation of the two groups) were color-coded and overlaid onto the mouse template. The positive $\mathrm{T}$ values indicate that the intensities in the first group are greater than in second group and color-coded from green to red.

\section{Figure 3}

Evaluation of the time course of the hippocampal volume change on MRI following pilocarpineinduced status epilepticus. Data are presented for the three groups: mice that experienced status epilepticus (SE group, $n=17$ ), mice without SE (NS group, $n=13$ ) and controls $(n=10)$. Note that there was a constant decrease in relative hippocampal volumes in the SE group, proposing progression of damage. Data are presented as mean \pm SD. ${ }^{*} p<0.05, * * p<0.01$ between the SE and NS groups. \#p<0.05, \#\#p<0.01, \#\#\#<0.001 between the SE and control groups. Abbreviations: $\mathrm{h}$, hours; d, days.

\section{Figure 4}

Correlation of MRI changes with epileptogenicity. (A) The relative ADC changes were correlated voxel-wise with the EEG results using spike frequency as a regressor. Scale: the significance level 
of $\mathrm{T}$ values (ratios of differences between the two groups to the standard deviation of the two groups) were color-coded and overlaid onto the mouse template. (B) A representative $10 \mathrm{sec}$ fragment of EEG showing interictal spikes (arrows) in 3 animals with epilepsy (traces highlighted in pink). No epileptiform changes were observed in NS (highlighted in blue) or control animals (white) during 3 weeks of video-EEG monitoring. Each animal is represented by 2 consecutive traces on EEG.

\section{Figure 5}

Immunostaining of C3 in mouse hippocampal tissue 48 hours after status epilepticus. (A) Double labelling of C3 (red) and NeuN (green) doesn not show any colocalization. Panel B shows increased expression observed in astrocytes - double labelling of C3 (red) and GFAP (green). (C) Double lebelling of C3 and Iba1 does not show increased C3 expression in activated microglia. Scale bar $100 \mu \mathrm{m}$. Abbreviations: SE, status epilepticus.

\section{Figure 6}

Immunostaining of C3 in mouse hippocampal tissue 7 days after status epilepticus. Similar to the expression at 48 hours post-SE, prominent C3 immunoreactivity was observed only in astrocytes double labelling of C3 (red) and GFAP (green), panel B. Scale bar $100 \mu \mathrm{m}$. Abbreviations: SE, status epilepticus.

\section{Figure 7}

Immunostaining of C3 in mouse hippocampal tissue 4 months after status epilepticus. Pronounced reactive astrocytosis can be observed in the subgranular zone of the dentate gyrus with astrocytes expressing C3 (panel B). Scale bar $100 \mu \mathrm{m}$. Abbreviations: SE, status epilepticus.

\section{Figure 8}

Distribution of C3 and GFAP immunoreactivity in the ventral hippocampus 7 days after pilocarpine-induced status epilepticus corresponds to the areas of MRI changes. (A) Significantly increased ADC values in the ventral hippocampus of SE animals compared to NS mice. (B) GFAP immunostaining of a representative animal from SE group. (C) Higher magnification of the hippocampal area in B. (D) Immunostaining of C3 in the hippocampal mouse tissue in a representative animal from SE group. (E,F) GFAP and C3 immunostaining of the corresponding ventral hippocampal area in a representative animal from NS group. Note prominent increased expression of $\mathrm{C} 3$ in astrocytes in the hilus and granule cell layer of the dentate gyrus, as well as ablation of CA1 in SE animal. Abbreviations: H, hilus; gcl, granule cell layer; CA1, Cornu Ammonis 1 region of the hippocampus proper Scale bar in C-F $100 \mu \mathrm{m}$.

\section{Figure 9}

Distribution of C3 immunoreactivity in the dorsal hippocampus 4 months after pilocarpineinduced status epilepticus. (A) Areas of significant ADC changes between SE and NS groups at 49 
days post-status epilepticus. (B) Immunostaining of $\mathrm{C} 3$ in the hippocampal tissue in a control animal. Control hippocampus shows weak $\mathrm{C} 3$ immunoreactivity in the hilar region and staining of blood vessels. (C1-3) Immunostaining of $\mathrm{C} 3$ in the hippocampal tissue from 3 representative animals without epilepsy (NS group). (D1-3) Immunostaining of C3 in the hippocampal tissue from 3 representative animals with spontaneous recurrent seizures (SE group). In non-epileptic animals weak $\mathrm{C} 3$ expression was found in the hilus and molecular layers of the dentate gyrus and around lumina of blood vessels. In animals with epilepsy prominent increased expression was observed in cells with typical morphology of reactive astrocytes in the subgranular zone and in the inner, middle and outer molecular layers of the dentate gyrus. Abbreviations: $\mathrm{CN}$, control animal; NS, animal without epilepsy; SE, animal with epilepsy; H, hilus; i, inner molecular layer; m, middle molecular layer; o, outer molecular layer; gcl, granule cell layer. Scale bar $100 \mu \mathrm{m}$.

\section{Table 1}

Summary of ADC SPM $\{t\}$ results between groups ${ }^{\S}$

${ }^{\S} \mathrm{AMG}, \mathrm{HC}, \mathrm{PIR}$, and PHC stand for the amygdala, hippocampus, piriform cortex, and parahippocampal cortex, respectively. SE, NS, and CN stand for status epilepticus, no seizures, and control groups, respectively. The $\operatorname{SPM}\{t\}$ results are presented as the estimated volume in $\mathrm{mm}^{3}$ (maximum T value) format. Example: SPM $\{\mathrm{t}\}$ of 2.15 (5.61) for AMG at 1 Day means that SPM found group difference in AMG with cluster size of $2.15 \mathrm{~mm}^{3}$ and maximum $\mathrm{T}$ value of 5.61. The $\mathrm{T}$ value represents the ratio of intensity differences between two groups to the standard deviation of two groups; negative $T$ value means the intensity in the first group is smaller than in the second group. If there are several clusters within a structure, the sum of the cluster volumes is reported. "“", no group difference detected by SPM. *Short-term changes in the thalamus included ADC drop 1Day post-SE - SPM $\{\mathrm{t}\} 5.98$ (-8.32), followed by increase 2 Days post-SE - SPM $\{\mathrm{t}\}$ 5.22 (5.78), see Fig.1B.

\section{Table 2}

Summary of T2 SPM $\{t\}$ results between groups ${ }^{\S}$

${ }^{\S} \mathrm{AMG}, \mathrm{HC}$, PIR, and PHC stand for the amygdala, hippocampus, piriform cortex, and parahippocampal cortex, respectively. SE, NS, and CN stand for status epilepticus, no seizures, and control groups, respectively. The SPM $\{t\}$ results are presented as the estimated volume in $\mathrm{mm}^{3}$ (maximum T value) format. Example: SPM $\{\mathrm{t}\}$ of 1.03 (5.19) for AMYG at 14 Day means that SPM found group difference in AMYG with cluster size of $1.03 \mathrm{~mm}^{3}$ and maximum $\mathrm{T}$ value of 5.19. The $\mathrm{T}$ value represents the ratio of intensity differences between two groups to the standard deviation of two groups; negative $\mathrm{T}$ value means the intensity in first group is smaller than in the second groups. If there are several clusters within a structure, the sum of the cluster volumes is reported. “-“, no group difference detected by SPM. 


\section{References}

Agster KL, Burwell RD (2009) Cortical efferents of the perirhinal, postrhinal, and entorhinal cortices of the rat. Hippocampus 19 (12):1159-1186. doi:10.1002/hipo.20578

Ang CW, Carlson GC, Coulter DA (2006) Massive and specific dysregulation of direct cortical input to the hippocampus in temporal lobe epilepsy. J Neurosci 26 (46):11850-11856. doi:10.1523/jneurosci.2354-06.2006

Aroniadou-Anderjaska V, Fritsch B, Qashu F, Braga MFM (2008) Pathology and pathophysiology of the amygdala in epileptogenesis and epilepsy. Epilepsy Res 78 (2-3):102-116. doi:10.1016/j.eplepsyres.2007.11.011

Aronica E, Boer K, van Vliet EA, Redeker S, Baayen JC, Spliet WGM, van Rijen PC, Troost D, da Silva FHL, Wadman WJ, Gorter JA (2007) Complement activation in experimental and human temporal lobe epilepsy. Neurobiol Dis 26 (3):497-511. doi:10.1016/j.nbd.2007.01.015

Avoli M, D'Antuono M, Louvel J, Kohling R, Biagini G, Pumain R, D'Arcangelo G, Tancredi V (2002) Network and pharmacological mechanisms leading to epileptiform synchronization in the limbic system in vitro. Prog Neurobiol 68 (3):167-207. doi:Pii s0301-0082(02)00077-1

Bartolomei F, Khalil M, Wendling F, Sontheimer A, Regis J, Ranjeva JP, Guye M, Chauvel P (2005) Entorhinal cortex involvement in human mesial temporal lobe epilepsy: An electrophysiologic and volumetric study. Epilepsia 46 (5):677-687. doi:10.1111/j.15281167.2005.43804.x

Benini R, Longo D, Biagini G, Avoli M (2011) Perirhinal cortex hyperexcitability in pilocarpinetreated epileptic rats. Hippocampus 21 (7):702-713. doi:10.1002/hipo.20785

Bernasconi N, Bernasconi A, Andermann F, Dubeau F, Feindel W, Reutens DC (1999) Entorhinal cortex in temporal lobe epilepsy - A quantitative MRI study. Neurology 52 (9):1870-1876

Bernasconi N, Bernasconi A, Caramanos Z, Andermann F, Dubeau F, Arnold DL (2000) Morphometric MRI Analysis of the Parahippocampal Region in Temporal Lobe Epilepsy. Ann N Y Acad Sci 911 (1):495-500. doi:10.1111/j.1749-6632.2000.tb06752.x

Bernasconi N, Bernasconi A, Caramanos Z, Antel SB, Andermann F, Arnold DL (2003) Mesial temporal damage in temporal lobe epilepsy: a volumetric MRI study of the hippocampus, amygdala and parahippocampal region. Brain 126:462-469. doi:10.1093/brain/awg034

Bernasconi N, Duchesne S, Janke A, Lerch J, Collins DL, Bernasconi A (2004) Whole-brain voxel-based statistical analysis of gray alter and white matter in temporal lobe epilepsy. Neuroimage 23 (2):717-723. doi:10.1016/j.neuroimage.2004.06.015

Bertram EH (1997) Functional anatomy of spontaneous seizures in a rat model of limbic epilepsy. Epilepsia 38 (1):95-105. doi:10.1111/j.1528-1157.1997.tb01083.x

Bertram EH (2009) Temporal lobe epilepsy: Where do the seizures really begin? Epilepsy Behav 14:32-37. doi:10.1016/j.yebeh.2008.09.017 
Bertram EH, Cornett JF (1994) The evolution of a rat model of chronic spontaneous limbic seizures. Brain Res 661 (1-2):157-162. doi:10.1016/0006-8993(94)91192-4

Bertram EH, Zhang DX, Mangan P, Fountain N, Rempe D (1998) Functional anatomy of limbic epilepsy: a proposal for central synchronization of a diffusely hyperexcitable network. Epilepsy Res 32 (1-2):194-205. doi:10.1016/s0920-1211(98)00051-5

Biagini G, D'Arcangelo G, Baldelli E, D'Antuono M, Tancredi V, Avoli M (2005) Impaired activation of CA3 pyramidal neurons in the epileptic hippocampus. Neuromolecular Med 7 (4):325-342. doi:10.1385/nmm:7:4:325

Bonilha L, Kobayashi E, Rorden C, Cendes F, Li LM (2003) Medial temporal lobe atrophy in patients with refractory temporal lobe epilepsy. J Neurol Neurosurg Psychiatry 74 (12):1627-1630. doi:10.1136/jnnp.74.12.1627

Bonilha L, Nesland T, Martz GU, Joseph JE, Spampinato MV, Edwards JC, Tabesh A (2012) Medial temporal lobe epilepsy is associated with neuronal fibre loss and paradoxical increase in structural connectivity of limbic structures. J Neurol Neurosurg Psychiatry 83 (9):903-909. doi:10.1136/jnnp-2012-302476

Borges K, Gearing M, McDermott DL, Smith AB, Almonte AG, Wainer BH, Dingledine R (2003) Neuronal and glial pathological changes during epileptogenesis in the mouse pilocarpine model. Exp Neurol 182 (1):21-34. doi:10.1016/s0014-4886(03)00086-4

Bragin A, Wilson CL, Engel J (2000) Chronic epileptogenesis requires development of a network of pathologically interconnected neuron clusters: A hypothesis. Epilepsia 41:S144-S152. doi:10.1111/j.1528-1157.2000.tb01573.x

Briellmann RS, Kalnins RM, Berkovic SF, Jackson GD (2002) Hippocampal pathology in refractory temporal lobe epilepsy - T2-weighted signal change reflects dentate gliosis. Neurology 58 (2):265-271

Buckmaster PS (2004) Laboratory animal models of temporal lobe epilepsy. Comparative Medicine 54 (5):473-485

Cavalheiro EA, Santos NF, Priel MR (1996) The pilocarpine model of epilepsy in mice. Epilepsia 37 (10):1015-1019. doi:10.1111/j.1528-1157.1996.tb00541.x

Chang BS, Lowenstein DH (2003) Mechanisms of disease - Epilepsy. New Engl J Med 349 (13):1257-1266. doi:10.1056/NEJMra022308

Chen J, Larionov S, Hoerold N, Ullmann C, Elger CE, Schramm J, Becker AJ (2005) Expression analysis of metabotropic glutamate receptors I and III in mouse strains with different susceptibility to experimental temporal lobe epilepsy. Neurosci Lett 375 (3):192-197. doi:10.1016/j.neulet.2004.11.008

Clemens Z, Janszky J, Clemens M, Szucs A, Halasz P (2005) Factors affecting spiking related to sleep and wake states in temporal lobe epilepsy (TLE). Seizure 14 (1):52-57. doi:10.1016/j.seizure.2004.09.003

Curia G, Longo D, Biagini G, Jones RSG, Avoli M (2008) The pilocarpine model of temporal lobe epilepsy. J Neurosci Methods 172 (2):143-157. doi:10.1016/j.jneumeth.2008.04.019 
De Guzman P, D'Antuono M, Avoli M (2004) Initiation of electrographic seizures by neuronal networks in entorhinal and perirhinal cortices in vitro. Neuroscience 123 (4):875-886. doi:10.1016/j.neuroscience.2003.11.013

Demir R, Haberly LB, Jackson MB (2000) Imaging epileptiform discharges in slices of piriform cortex with voltage-sensitive fluorescent dyes. Ann N Y Acad Sci 911:404-417

Demir R, Haberly LB, Jackson MB (2001) Epileptiform discharges with in-vivo-like features in slices of rat piriform cortex with longitudinal association fibers. J Neurophysiol 86 (5):2445-2460

Dudek FE, Staley KJ (2011) The time course of acquired epilepsy: Implications for therapeutic intervention to suppress epileptogenesis. Neurosci Lett 497 (3):240-246. doi:10.1016/j.neulet.2011.03.071

Dudek FE, Sutula TP (2007) Epileptogenesis in the dentate gyrus: a critical perspective. Prog Brain Res 163:755-773. doi:10.1016/s0079-6123(07)63041-6

Engel J, Jr. (2011) Biomarkers in epilepsy: introduction. Biomarkers Med 5 (5):537-544. doi:10.2217/bmm.11.62

Engel J, Williamson PD, Wieser HG (2008) Mesial temporal lobe epilepsy with hippocampal sclerosis. In: Engel J, Pedley TA (eds) Epilepsy: A Comprehensive Textbook. 2nd edn. Lippincott-Raven, Philadelphia, pp 2479-2486

Fabene PF, Marzola P, Sbarbati A, Bentivoglio M (2003) Magnetic resonance imaging of changes elicited by status epilepticus in the rat brain: diffusion-weighted and T2-weighted images, regional blood volume maps, and direct correlation with tissue and cell damage. Neuroimage 18 (2):375-389. doi:Pii s1053-8119(02)00025-3

Fabene PF, Weiczner R, Marzola P, Nicolato E, Calderan L, Andrioli A, Farkas E, Sule Z, Mihaly A, Sbarbati A (2006) Structural and functional MRI following 4-aminopyridine-induced seizures: A comparative imaging and anatomical study. Neurobiol Dis 21 (1):80-89. doi:10.1016/j.nbd.2005.06.013

Feindel W, Rasmussen T (1991) Temporal lobectomy with amygdalectomy and minimal hippocampal resection - review of 100 cases. Can J Neurol Sci 18 (4):603-605

French JA, Williamson PD, Thadani VM, Darcey TM, Mattson RH, Spencer SS, Spencer DD (1993) Characteristics of medial temporal lobe epilepsy -. I. Results of history and physical examination. Ann Neurol 34 (6):774-780. doi:10.1002/ana.410340604

Giblin KA, Blumenfeld H (2010) Is epilepsy a preventable disorder? New evidence from animal models. Neuroscientist 16 (3):253-275. doi:10.1177/1073858409354385

Halonen T, Tortorella A, Zrebeet H, Gale K (1994) Posterior piriform and perirhinal cortex relay seizures evoked from the area tempestas - role of excitatory and inhibitory amino acid receptors. Brain Res 652 (1):145-148. doi:10.1016/0006-8993(94)90328-x

Herman ST (2006) Clinical trials for prevention of epileptogenesis. Epilepsy Res 68 (1):35-38. doi:10.1016/j.eplepsyres.2005.09.015

Jackson GD, Berkovic SF, Duncan JS, Connelly A (1993) Optimizing the diagnosis of hippocampal sclerosis using MR imaging. Am J Neuroradiol 14 (3):753-762 
Jackson GD, Berkovic SF, Tress BM, Kalnins RM, Fabinyi GCA, Bladin PF (1990) Hippocampal sclerosis can be reliably detected by magnetic-resonance-imaging. Neurology 40 (12):1869-1875

Jamali S, Bartolomei F, Robaglia-Schlupp A, Massacrier A, Peragut JC, Regis J, Dufour H, Ravid R, Roll P, Pereira S, Royer B, Roeckel-Trevisiol N, Fontaine M, Guye M, Boucraut J, Chauvel P, Cau P, Szepetowski P (2006) Large-scale expression study of human mesial temporal lobe epilepsy: evidence for dysregulation of the neurotransmission and complement systems in the entorhinal cortex. Brain 129:625-641.

doi:10.1093/brain/awl001

Jamali S, Salzmann A, Perroud N, Ponsole-Lenfant M, Cillario J, Roll P, Roeckel-Trevisiol N, Crespel A, Balzar J, Schlachter K, Gruber-Sedlmayr U, Pataraia E, Baumgartner C, Zimprich A, Zimprich F, Malafosse A, Szepetowski P (2010) Functional variant in complement $\mathrm{C} 3$ gene promoter and genetic susceptibility to temporal lobe epilepsy and febrile seizures. Plos One 5 (9):e12740. doi:10.1371/journal.pone.0012740

Janszky J, Hoppe M, Clemens Z, Janszky I, Gyimesi C, Schulz R, Ebner A (2005) Spike frequency is dependent on epilepsy duration and seizure frequency in temporal lobe epilepsy. Epileptic Disord 7 (4):355-359

Jooma R, Yeh HS, Privitera MD, Rigrish D, Gartner M (1995) Seizure control and extent of mesial temporal resection. Acta Neurochir 133 (1-2):44-49. doi:10.1007/bf01404946

Keller SS, Mackay CE, Barrick TR, Wieshmann UC, Howard MA, Roberts N (2002) Voxel-based morphometric comparison of hippocampal and extrahippocampal abnormalities in patients with left and right hippocampal atrophy. Neuroimage 16 (1):23-31. doi:10.1006/nimg.2001.1072

Keller SS, Wilke M, Wieshmann UC, Sluming VA, Roberts N (2004) Comparison of standard and optimized voxel-based morphometry for analysis of brain changes associated with temporal lobe epilepsy. Neuroimage 23 (3):860-868. doi:10.1016/j.neuroimage.2004.07.030

Kelly ME, McIntyre DC (1996) Perirhinal cortex involvement in limbic kindled seizures. Epilepsy Res 26 (1):233-243. doi:10.1016/s0920-1211(96)00056-3

Kharatishvili I, Immonen R, Grohn O, Pitkanen A (2007) Quantitative diffusion MRI of hippocampus as a surrogate marker for post-traumatic epileptogenesis. Brain 130:31553168. doi:10.1093/brain/awm268

Krendl R, Lurger S, Baumgartner C (2008) Absolute spike frequency predicts surgical outcome in TLE with unilateral hippocampal atrophy. Neurology 71 (6):413-418. doi:10.1212/01.wnl.0000310775.87331.90

Kuzniecky RI, Bilir E, Gilliam F, Faught E, Palmer C, Morawetz R, Jackson G (1997) Multimodality MRI in mesial temporal sclerosis: Relative sensitivity and specificity. Neurology 49 (3):774-778

Libbey JE, Kirkman NJ, Wilcox KS, White HS, Fujinami RS (2010) Role for complement in the development of seizures following acute viral infection. J Virol 84 (13):6452-6460. doi:10.1128/jvi.00422-10 
Loscher W, Ebert U (1996) The role of the piriform cortex in kindling. Prog Neurobiol 50 (56):427-481. doi:10.1016/s0301-0082(96)00036-6

Lucas SM, Rothwell NJ, Gibson RM (2006) The role of inflammation in CNS injury and disease. Brit J Pharmacol 147:S232-S240. doi:10.1038/sj.bjp.0706400

Marchi N, Fan Q, Ghosh C, Fazio V, Bertolini F, Betto G, Batra A, Carlton E, Najm I, Granata T, Janigro D (2009) Antagonism of peripheral inflammation reduces the severity of status epilepticus. Neurobiol Dis 33 (2):171-181. doi:10.1016/j.nbd.2008.10.002

Marchi N, Granata T, Freri E, Ciusani E, Ragona F, Puvenna V, Teng Q, Alexopolous A, Janigro D (2011) Efficacy of anti-Inflammatory therapy in a model of acute seizures and in a population of pediatric drug resistant epileptics. Plos One 6 (3):e18200. doi:10.1371/journal.pone.0018200

Mathern GW, Babb TL, Vickrey BG, Melendez M, Pretorius JK (1995) The clinical-pathogenic mechanisms of hippocampal neuron loss and surgical outcomes in temporal lobe epilepsy. Brain 118:105-118. doi:10.1093/brain/118.1.105

McDonald CR, Hagler DJ, Jr., Ahmadi ME, Tecoma E, Iragui V, Gharapetian L, Dale AM, Halgren E (2008) Regional neocortical thinning in mesial temporal lobe epilepsy. Epilepsia 49 (5):794-803. doi:10.1111/j.1528-1167.2008.01539.x

McIntyre DC, Gilby KL (2006) Parahippocampal networks, intractability, and the chronic epilepsy of kindling. Adv Neurol 97:77-83

McIntyre DC, Gilby KL (2008) Mapping seizure pathways in the temporal lobe. Epilepsia 49:2330. doi:10.1111/j.1528-1167.2008.01507.x

McIntyre DC, Kelly ME (2000) The parahippocampal cortices and kindling. Ann N Y Acad Sci 911:343-354

McIntyre DC, Kelly ME, Armstrong JN (1993) Kindling in the perirhinal cortex. Brain Res 615 (1):1-6. doi:10.1016/0006-8993(93)91108-5

McNamara JO (1994) Cellular and molecular basis of epilepsy. J Neurosci 14 (6):3413-3425

Meade CE, Bowden SC, Whelan G, Cook MJ (2008) Rhinal cortex asymmetries in patients with mesial temporal sclerosis. Seizure 17 (3):234-246. doi:10.1016/j.seizure.2007.07.010

Mueller CJ, Bankstahl M, Groeticke I, Loescher W (2009) Pilocarpine vs. lithium-pilocarpine for induction of status epilepticus in mice: Development of spontaneous seizures, behavioral alterations and neuronal damage. Eur J Pharmacol 619 (1-3):15-24.

doi:10.1016/j.ejphar.2009.07.020

Nakasu Y, Nakasu S, Morikawa S, Uemura S, Inubushi T, Handa J (1995) Diffusion-weighted MR in experimental sustained seizures elicited with kainic acid. Am J Neuroradiol 16 (6):1185-1192

Natsume J, Bernasconi N, Andermann F, Bernasconi A (2003) MRI volumetry of the thalamus in temporal, extratemporal, and idiopathic generalized epilepsy. Neurology 60 (8):12961300

Nehlig A (2011) Hippocampal MRI and other structural biomarkers: experimental approach to epileptogenesis. Biomarkers Med 5 (5):585-597. doi:10.2217/bmm.11.65 
Parekh MB, Carney PR, Sepulveda H, Norman W, King M, Mareci TH (2010) Early MR diffusion and relaxation changes in the parahippocampal gyrus precede the onset of spontaneous seizures in an animal model of chronic limbic epilepsy. Exp Neurol 224 (1):258-270. doi:10.1016/j.expneurol.2010.03.031

Paxinos G, Franklin KBJ (2001) The mouse brain In stereotaxic coordinates. Academic Press, New York

Pell GS, Briellmann RS, Waites AB, Abbott DF, Jackson GD (2004) Voxel-based relaxometry: a new approach for analysis of T2 relaxometry changes in epilepsy. Neuroimage 21 (2):707-713. doi:10.1016/j.neuroimage.2003.09.059

Pereira PMG, Insausti R, Artacho-Perula E, Salmenpera T, Kalviainen R, Pitkanen A (2005) MR volumetric analysis of the piriform cortex and cortical amygdala in drug-refractory temporal lobe epilepsy. Am J Neuroradiol 26 (2):319-332

Pikkarainen M, Pitkanen A (2001) Projections from the lateral, basal and accessory basal nuclei of the amygdala to the perirhinal and postrhinal cortices in rat. Cereb Cortex 11 (11):10641082. doi:10.1093/cercor/11.11.1064

Pitkanen A (2010) Therapeutic approaches to epileptogenesis-Hope on the horizon. Epilepsia 51:2-17. doi:10.1111/j.1528-1167.2010.02602.x

Pitkanen A, Lukasiuk K (2011) Mechanisms of epileptogenesis and potential treatment targets. Lancet Neurol 10 (2):173-186. doi:10.1016/s1474-4422(10)70310-0

Pitkanen A, Sutula TP (2002) Is epilepsy a progressive disorder? Prospects for new therapeutic approaches in temporal-lobe epilepsy. Lancet Neurol 1 (3):173-181. doi:10.1016/s14744422(02)00073-X

Pitkanen A, Tuunanen J, Kalviainen R, Partanen K, Salmenpera T (1998) Amygdala damage in experimental and human temporal lobe epilepsy. Epilepsy Res 32 (1-2):233-253. doi:10.1016/s0920-1211(98)00055-2

Racine RJ (1972) Modification of seizure activity by electrical stimulation.2. Motor seizure. Electroencephalogr Clin Neurophysiol 32 (3):281-294. doi:10.1016/00134694(72)90177-0

Rakhade SN, Jensen FE (2009) Epileptogenesis in the immature brain: emerging mechanisms. Nat Rev Neurol 5 (7):380-391. doi:10.1038/nrneurol.2009.80

Roch C, Leroy C, Nehlig A, Namer IJ (2002) Predictive value of cortical injury for the development of temporal lobe epilepsy in 21-day-old rats: An MRI approach using the lithium-pilocarpine model. Epilepsia 43 (10):1129-1136. doi:10.1046/j.15281157.2002.17802.x

Rosati A, Aghakhani Y, Bernasconi A, Olivier A, Andermarm F, Gotman J, Dubeau F (2003) Intractable temporal lobe epilepsy with rare spikes is less severe than with frequent spikes. Neurology 60 (8):1290-1295

Schwarcz R, Bertram E, Scharfman HE (2002) Temporal lobe epilepsy: renewed emphasis on extrahippocampal areas. In: Davis KL, Charney D, Coyle JT, Nemeroff C (eds) Neuropsychopharmacology: Fifth Generation of Progress. 5th edn. American College of Neuropsychopharmacology, Tennessee, pp 1844-1857 
Shan ZY, Ji Q, Gajjar A, Reddick WE (2005) A knowledge-guided active contour method of segmentation of cerebella on MR images of pediatric patients with medulloblastoma. J Magn Reson Im 21 (1):1-11. doi:10.1002/jmri.20229

Sharma AK, Reams RY, Jordan WH, Miller MA, Thacker HL, Snyder PW (2007) Mesial temporal lobe epilepsy: Pathogenesis, induced rodent models and lesions. Toxicol Pathol 35 (7):984-999. doi:10.1080/01926230701748305

Shibley H, Smith BN (2002) Pilocarpine-induced status epilepticus results in mossy fiber sprouting and spontaneous seizures in C57BL/6 and CD-1 mice. Epilepsy Res 49 (2):109120. doi:10.1016/s0920-1211(02)00012-8

Sloviter RS, Bumanglag AV (2012) Defining “epileptogenesis" and identifying "antiepileptogenic targets" in animal models of acquired temporal lobe epilepsy is not as simple as it might seem. Neuropharmacology Epub. doi:10.1016/j.neuropharm.2012.01.022

Spencer SS, Spencer DD (1994) Entorhinal-hippocampal interactions in medial temporal lobe epilepsy. Epilepsia 35 (4):721-727. doi:10.1111/j.1528-1157.1994.tb02502.x

Tuunanen J, Halonen T, Pitkanen A (1996) Status epilepticus causes selective regional damage and loss of GABAergic neurons in the rat amygdaloid complex. Eur J Neurosci 8 (12):2711-2725. doi:10.1111/j.1460-9568.1996.tb01566.x

Tuunanen J, Halonen T, Pitkanen A (1997) Decrease in somatostatin-immunoreactive neurons in the rat amygdaloid complex in a kindling model of temporal lobe epilepsy. Epilepsy Res 26 (2):315-327. doi:10.1016/s0920-1211(96)00900-х

Ullmann JFP, Keller MD, Watson C, Janke AL, Kurniawan ND, Yang Z, Richards K, Paxinos G, Egan GF, Petrou S, Bartlett P, Galloway GJ, Reutens DC (2012) Segmentation of the C57BL/6J mouse cerebellum in magnetic resonance images. Neuroimage 62 (3):14081414. doi:10.1016/j.neuroimage.2012.05.061

VanLandingham KE, Heinz ER, Cavazos JE, Lewis DV (1998) Magnetic resonance imaging evidence of hippocampal injury after prolonged focal febrile convulsions. Ann Neurol 43 (4):413-426. doi:10.1002/ana.410430403

Vezzani A, Aronica E, Mazarati A, Pittman QJ (2011a) Epilepsy and brain inflammation. Exp Neurol Epub. doi:10.1016/j.expneurol.2011.09.033

Vezzani A, French J, Bartfai T, Baram TZ (2011b) The role of inflammation in epilepsy. Nat Rev Neurol 7 (1):31-40. doi:10.1038/nrneurol.2010.178

Vezzani A, Granata T (2005) Brain inflammation in epilepsy: Experimental and clinical evidence. Epilepsia 46 (11):1724-1743. doi:10.1111/j.1528-1167.2005.00298.x

Vezzani A, Maroso M, Balosso S, Sanchez M-A, Bartfai T (2011c) IL-1 receptor/Toll-like receptor signaling in infection, inflammation, stress and neurodegeneration couples hyperexcitability and seizures. Brain Behav Immun 25 (7):1281-1289. doi:10.1016/j.bbi.2011.03.018

Vezzani A, Ravizza T, Balosso S, Aronica E (2008) Glia as a source of cytokines: Implications for neuronal excitability and survival. Epilepsia 49:24-32. doi:10.1111/j.1528-

1167.2008.01490.x 
Walker MC, White HS, Sander J (2002) Disease modification in partial epilepsy. Brain 125:19371950. doi:10.1093/brain/awf203

Wall CJ, Kendall EJ, Obenaus A (2000) Rapid alterations in diffusion-weighted images with anatomic correlates in a rodent model of status epilepticus. Am J Neuroradiol 21 (10):1841-1852

Wang Y, Majors A, Najm I, Xue M, Comair Y, Modic M, Ng TC (1996) Postictal alteration of sodium content and apparent diffusion coefficient in epileptic rat brain induced by kainic acid. Epilepsia 37 (10):1000-1006. doi:10.1111/j.1528-1157.1996.tb00539.x

White HS (2002) Animal models of epileptogenesis. Neurology 59 (9):S7-S14

White LE, Price JL (1993a) The functional anatomy of limbic status epilepticus in the rat. 1. Patterns of 14C-2-deoxyglucose uptake and Fos immunocytochemistry. J Neurosci 13 (11):4787-4809

White LE, Price JL (1993b) The functional anatomy of limbic status epilepticus in the rat. 2. The effects of focal deactivation. J Neurosci 13 (11):4810-4830

Wieser HG (2000) Mesial temporal lobe epilepsy versus amygdalar epilepsy: late seizure recurrence after initially successful amygdalotomy and regained seizure control following hippocampectomy. Epileptic Disord 2 (3):141-151

Winawer MR, Makarenko N, McCloskey DP, Hintz TM, Nair N, Palmer AA, Scharfman HE (2007) Acute and chronic responses to the convulsant pilocarpine in DBA/2J and A/J mice. Neuroscience 149 (2):465-475. doi:10.1016/j.neuroscience.2007.06.009

Wuarin JP, Dudek FE (1996) Electrographic seizures and new recurrent excitatory circuits in the dentate gyrus of hippocampal slices from kainate-treated epileptic rats. J Neurosci 16 (14):4438-4448

Wuarin JP, Dudek FE (2001) Excitatory synaptic input to granule cells increases with time after kainate treatment. J Neurophysiol 85 (3):1067-1077 
Table I Summary of ADC SPM $\{t\}$ results between groups ${ }^{\S}$

\begin{tabular}{|c|c|c|c|c|c|c|c|c|c|c|c|c|}
\hline \multirow{2}{*}{ Times } & \multicolumn{4}{|c|}{$\mathrm{SE}-\mathrm{CN}^{*}$} & \multicolumn{4}{|c|}{ SE - NS } & \multicolumn{4}{|c|}{$\mathrm{NS}-\mathrm{CN}$} \\
\hline & $\mathrm{AMG}$ & $\mathrm{HC}$ & PIR & $\mathrm{PHC}$ & $\mathrm{AMG}$ & $\mathrm{HC}$ & PIR & PHC & $\mathrm{AMG}$ & $\mathrm{HC}$ & PIR & PHC \\
\hline $3 \mathrm{~h}$ & - & - & - & - & - & - & - & - & - & - & - & - \\
\hline $1 \mathrm{D}$ & $2.15(5.61)$ & $1.02(-8.32)$ & - & $4.28(5.13)$ & - & - & - & - & $0.56(4.44)$ & - & - & - \\
\hline $2 \mathrm{D}$ & $4.56(7.21)$ & $2.9(7.11)$ & - & $4.63(5.76)$ & $3.56(7.21)$ & $1.6(4.3)$ & $3.87(6.21)$ & $2.57(5.76)$ & $1.86(7.21)$ & $0.48(4.5)$ & - & - \\
\hline $7 \mathrm{D}$ & $1.73(6.86)$ & $3.47(7.22)$ & $2.13(5.76)$ & $2.34(6.74)$ & $2.08(7.11)$ & $1.78(7.31)$ & $4.12(6.52)$ & - & - & $0.78(7.22)$ & - & - \\
\hline $14 \mathrm{D}$ & $4.03(7.31)$ & $4.03(7.11)$ & $3.78(5.83)$ & $4.19(5.92)$ & $4.08(5.61)$ & $1.38(4.31)$ & - & $3.13(4.67)$ & - & - & - & - \\
\hline $28 \mathrm{D}$ & $2.03(7.2)$ & $1.98(7.09)$ & $1.26(6.25)$ & - & $4.08(5.61)$ & $1.88(7.31)$ & - & - & - & - & - & - \\
\hline $35 \mathrm{D}$ & - & $2.03(5.62)$ & - & $5.81(5.97)$ & - & $0.78(6.81)$ & - & $1.06(5.96)$ & - & - & - & - \\
\hline 49D & - & 2.08 (7.19) & - & - & - & $1.78(7.23)$ & - & - & - & - & - & - \\
\hline
\end{tabular}

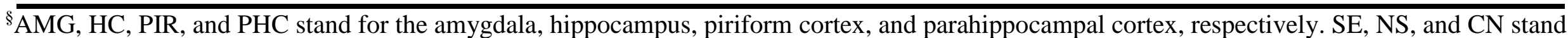
for status epilepticus, no seizures, and control groups, respectively. 
Table II Summary of T2 SPM $\{t\}$ results between groups ${ }^{\S}$

\begin{tabular}{|c|c|c|c|c|c|c|c|c|c|c|c|c|}
\hline \multirow{2}{*}{ Times } & \multicolumn{4}{|c|}{$\mathrm{SE}-\mathrm{CN}$} & \multicolumn{4}{|c|}{ SE - NS } & \multicolumn{4}{|c|}{$\mathrm{NS}-\mathrm{CN}$} \\
\hline & $\mathrm{AMG}$ & $\mathrm{HC}$ & PIR & PHC & $\mathrm{AMG}$ & $\mathrm{HC}$ & PIR & PHC & AMG & $\mathrm{HC}$ & PIR & PHC \\
\hline $3 \mathrm{~h}$ & - & - & - & - & - & - & - & - & - & - & - & - \\
\hline $1 \mathrm{D}$ & - & - & - & - & - & - & - & - & - & - & - & - \\
\hline $2 \mathrm{D}$ & - & $3.52(5.39)$ & - & - & $1.26(5.75)$ & - & - & - & $0.75(5.81)$ & $1.23(5.75)$ & - & - \\
\hline $7 \mathrm{D}$ & - & $3.49(5.38)$ & - & - & $2.88(5.85)$ & $1.77(4.24)$ & $1.26(4.32)$ & - & - & - & - & - \\
\hline $14 \mathrm{D}$ & $1.03(5.19)$ & $3.89(5.4)$ & - & $1.06(5.22)$ & $1.98(5.76)$ & $0.87(4.2)$ & $0.93(5.76)$ & $1.45(4.93)$ & - & - & - & - \\
\hline $28 \mathrm{D}$ & $2.03(5.41)$ & $1.97(4.24)$ & - & - & - & $0.67(4.2)$ & - & - & - & - & - & - \\
\hline $35 \mathrm{D}$ & $1.63(5.35)$ & $4.2(5.38)$ & $2.21(5.25)$ & $2.91(4.99)$ & $1.68(5.81)$ & $0.97(4.32)$ & - & $1.56(5.59)$ & - & - & - & - \\
\hline 49D & $1.53(5.28)$ & $3.89(4.28)$ & - & - & $2.68(5.21)$ & $1.17(4.2)$ & $6.21(5.09)$ & - & - & - & - & - \\
\hline
\end{tabular}

$\S \overline{A M G, ~ H C, ~ P I R, ~ a n d ~ P H C ~ s t a n d ~ f o r ~ t h e ~ a m y g d a l a, ~ h i p p o c a m p u s, ~ p i r i f o r m ~ c o r t e x, ~ a n d ~ p a r a h i p p o c a m p a l ~ c o r t e x, ~ r e s p e c t i v e l y . ~ S E, ~ N S, ~ a n d ~ C N ~}$ stand for status epilepticus, no seizures, and control groups, respectively. 


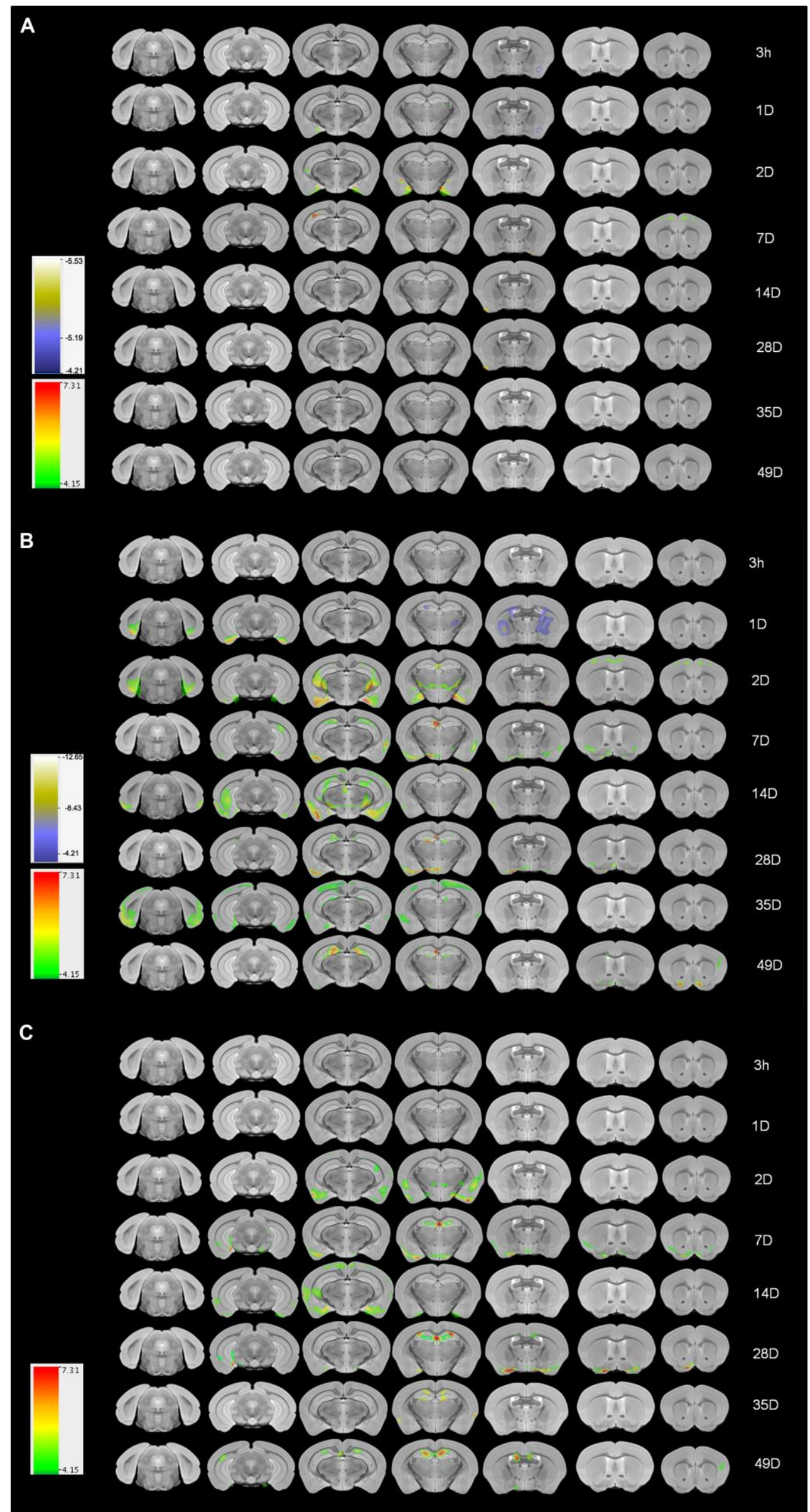

Fig 1 


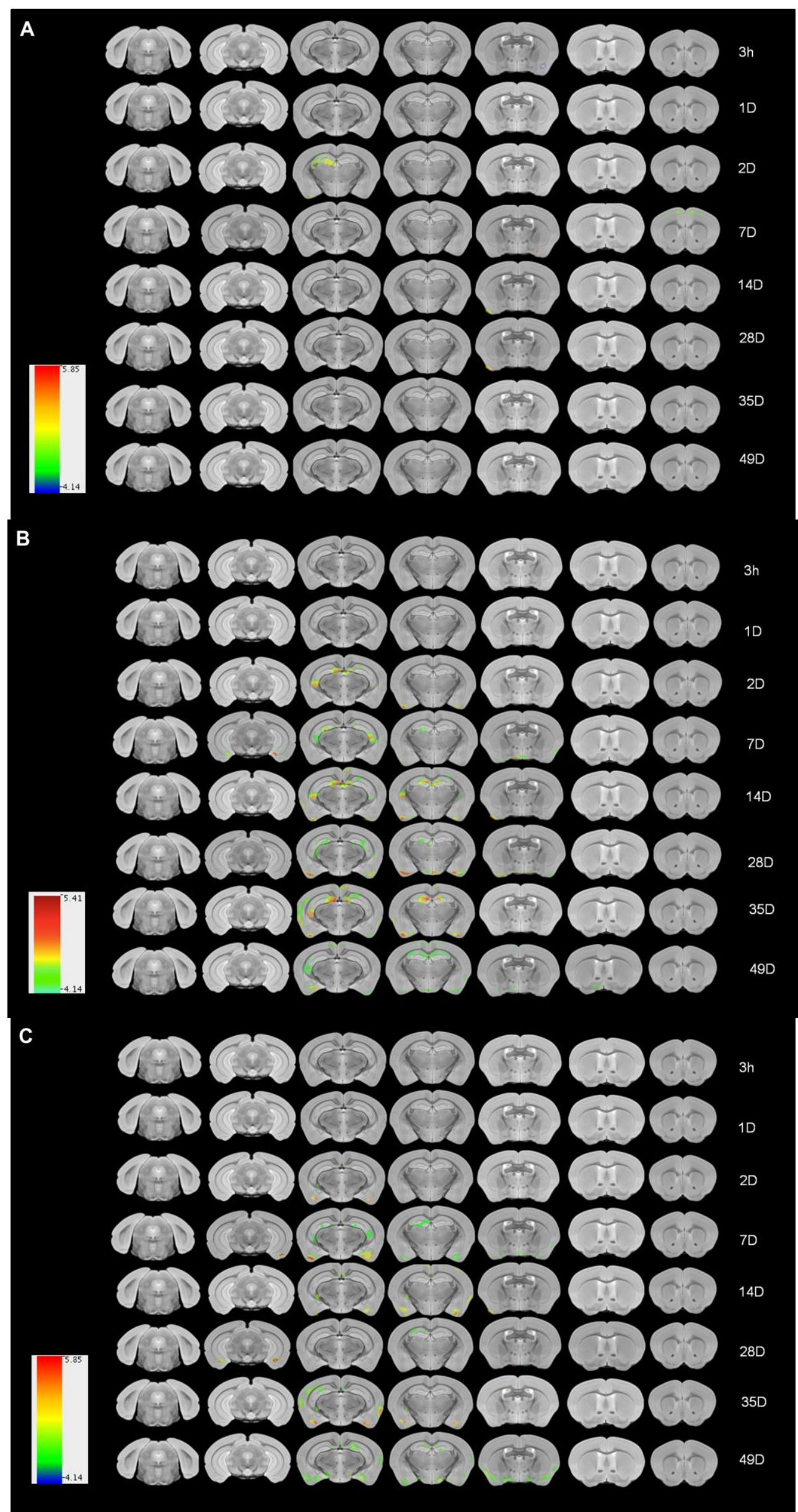

Fig. 2 

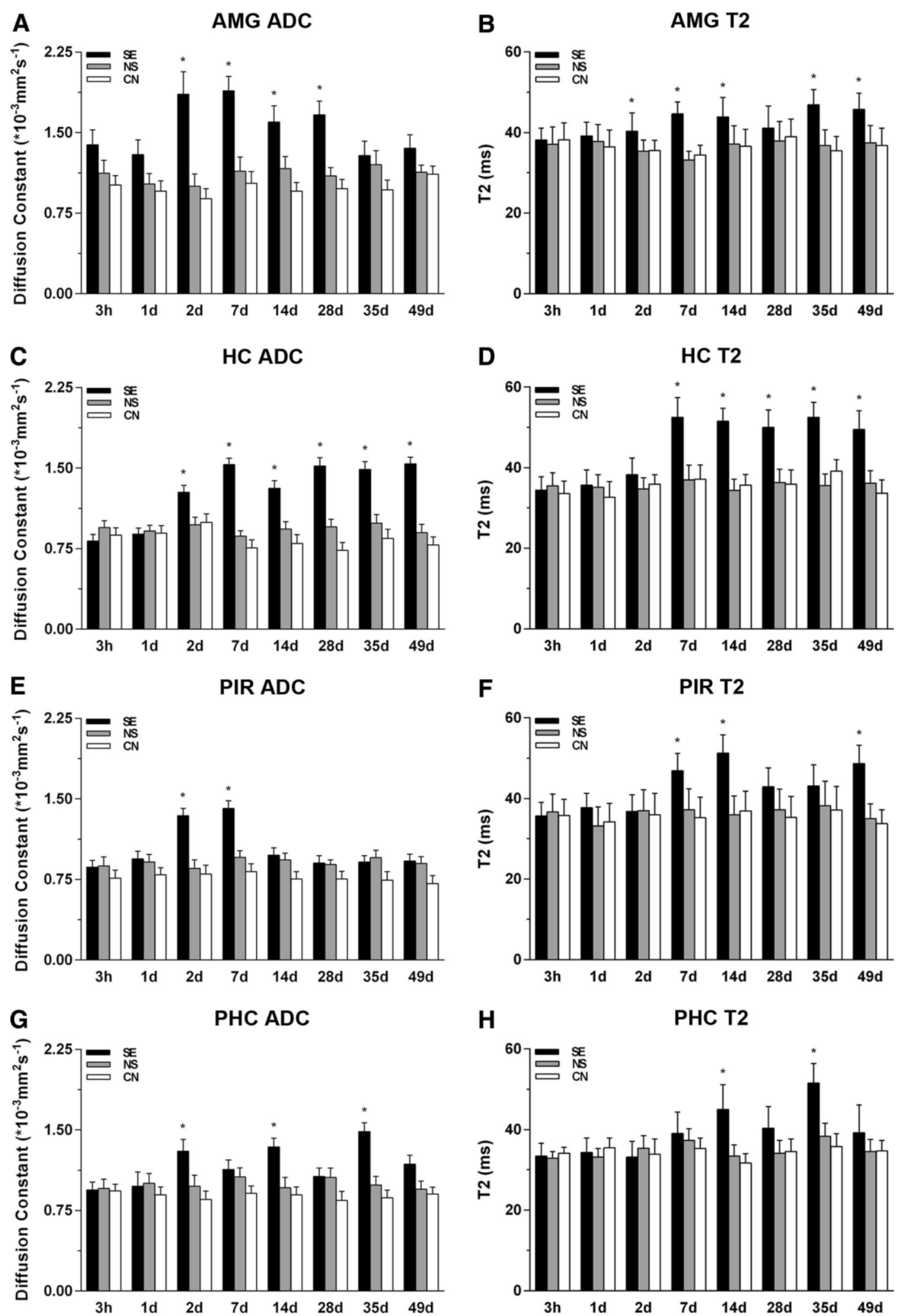

Fig. 3 


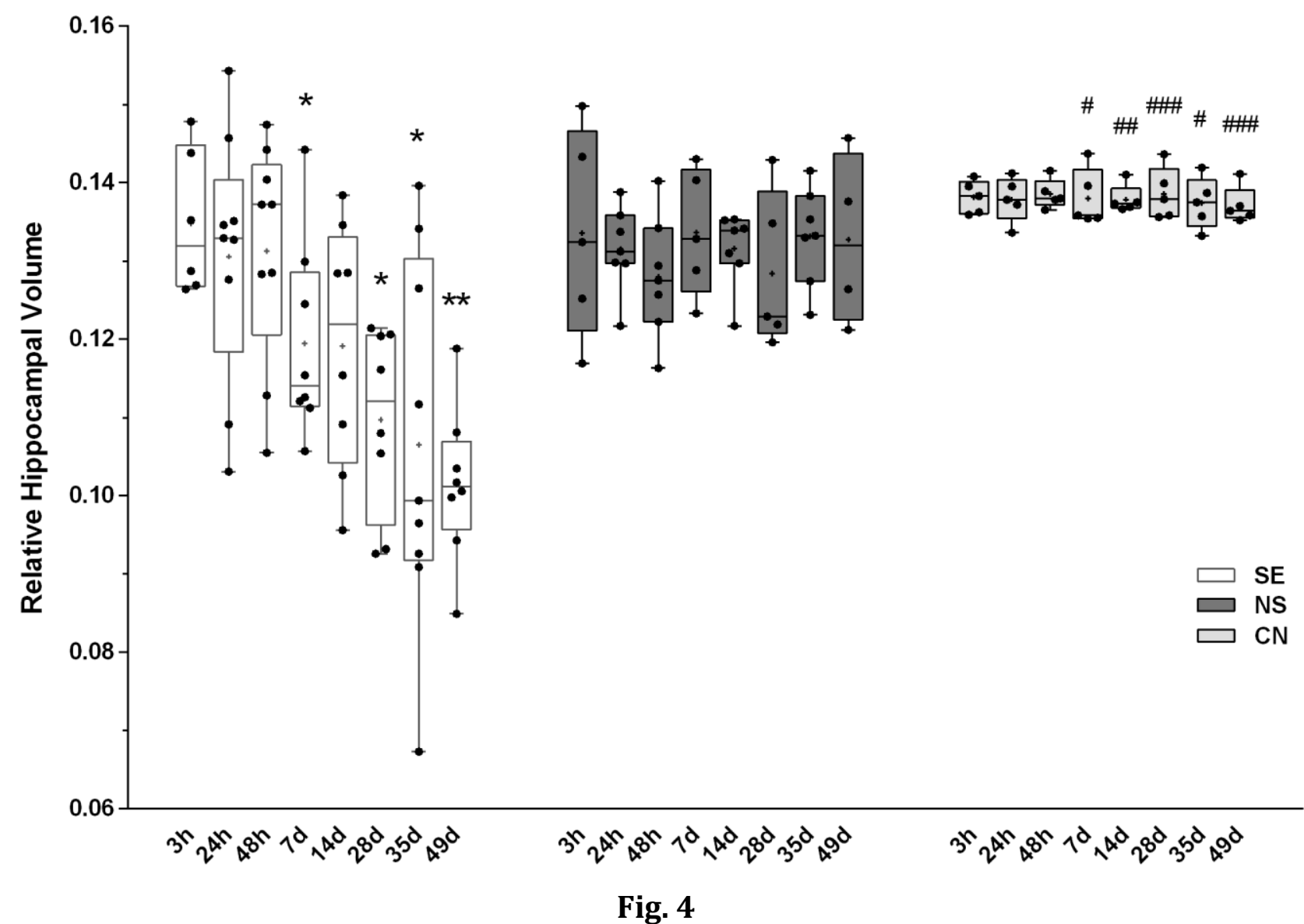




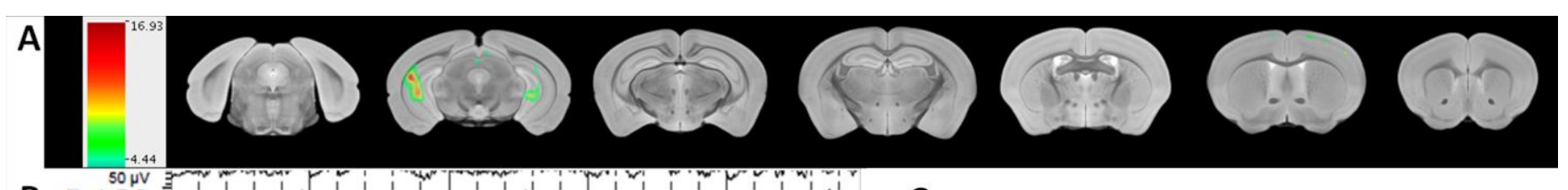

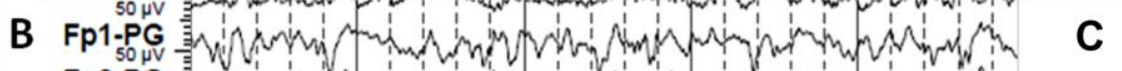

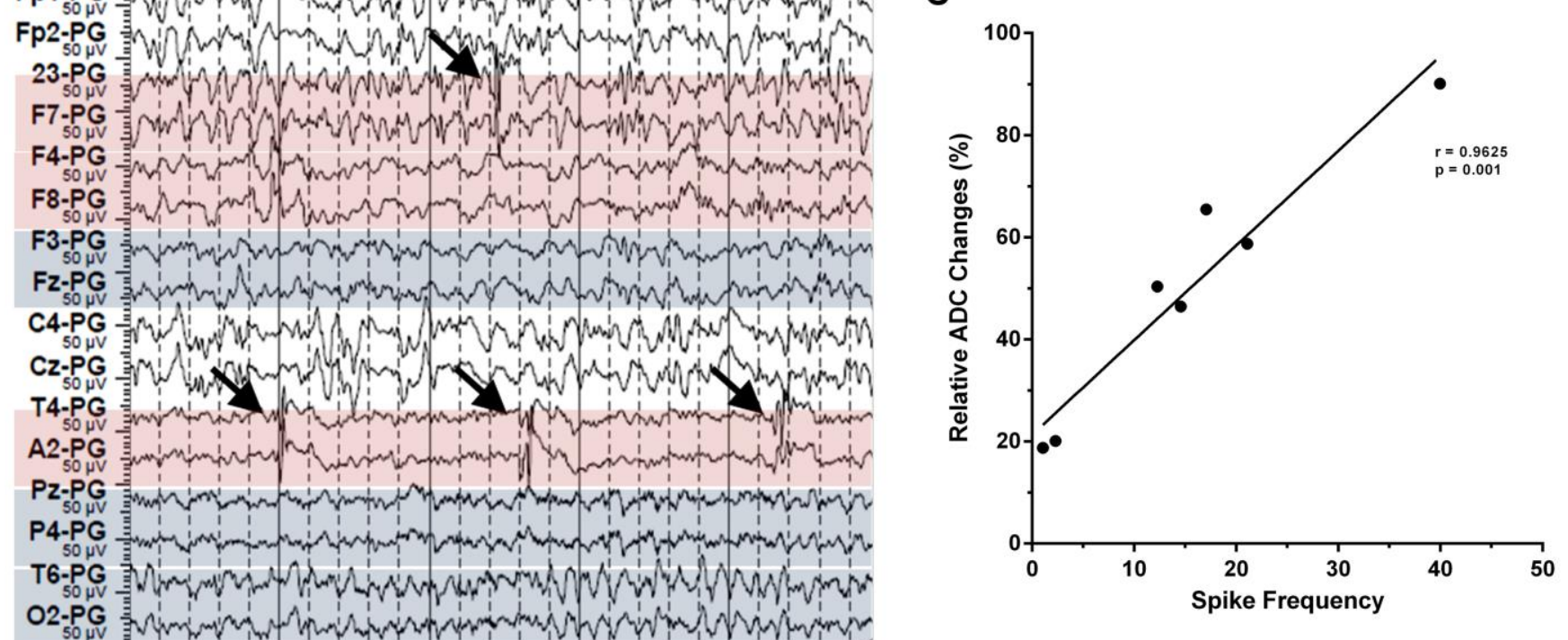

Fig. 5 


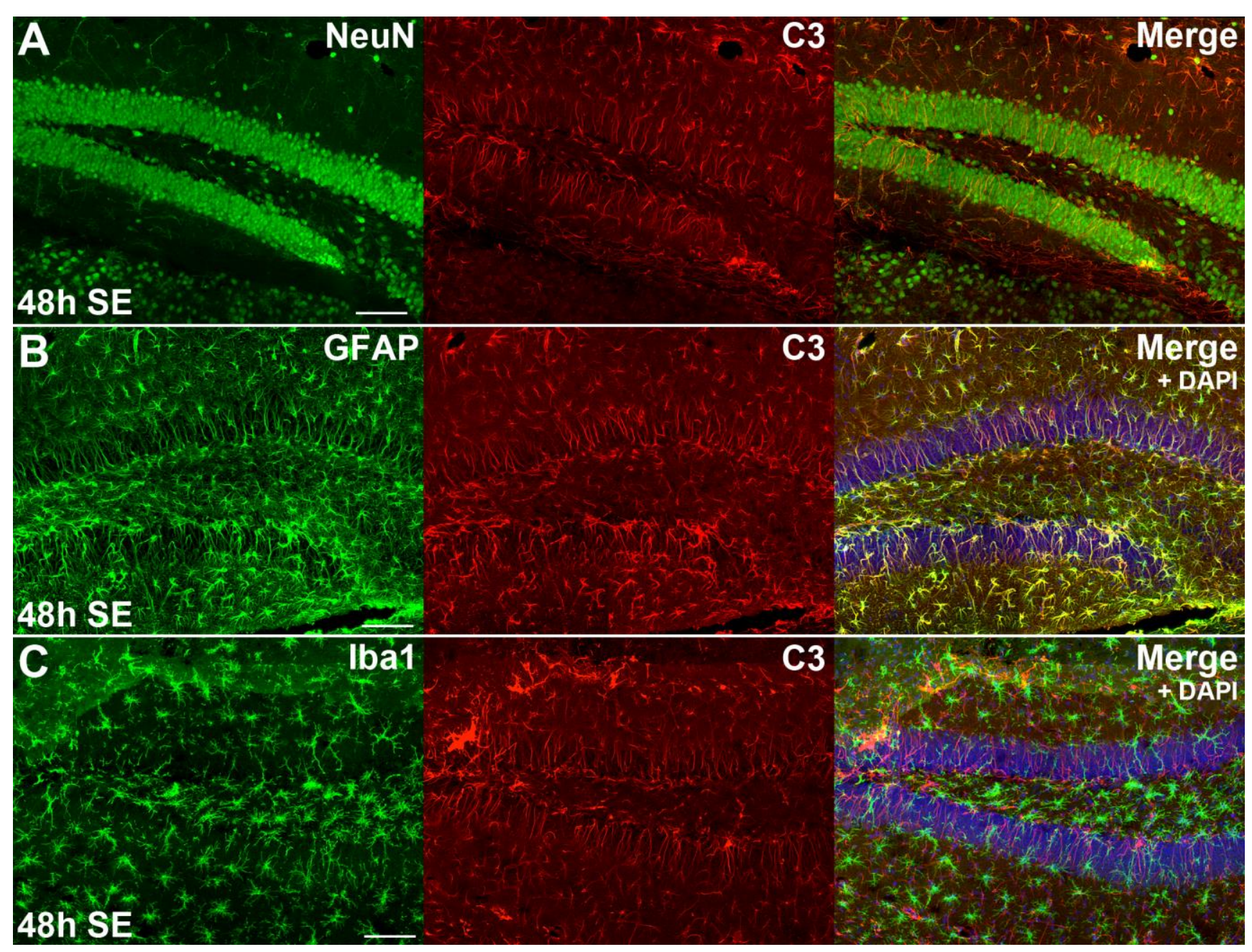

Fig. 6 


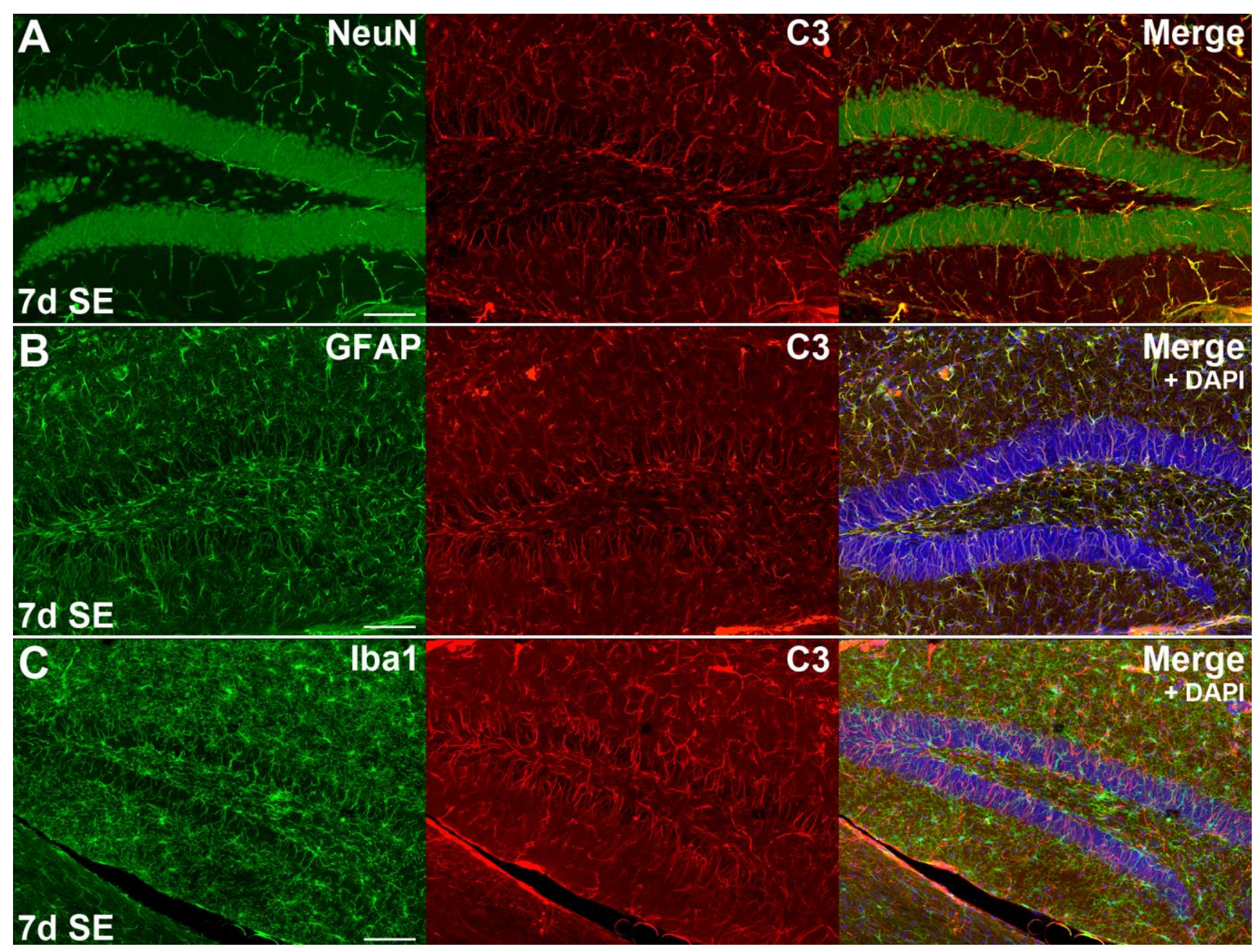

Fig. 7 


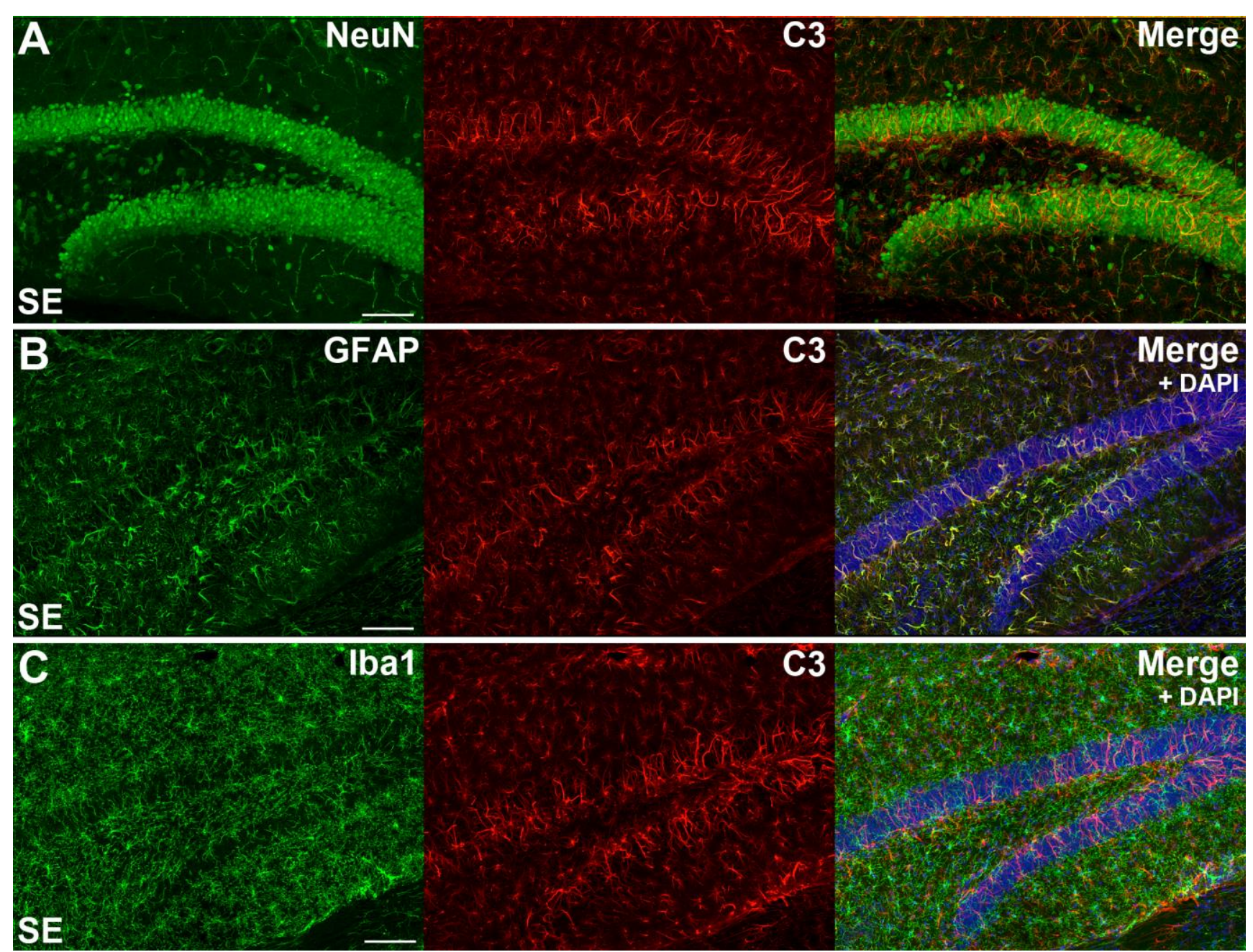

Fig. 8 
Page $\mathbf{3 9}$ of $\mathbf{4 2}$

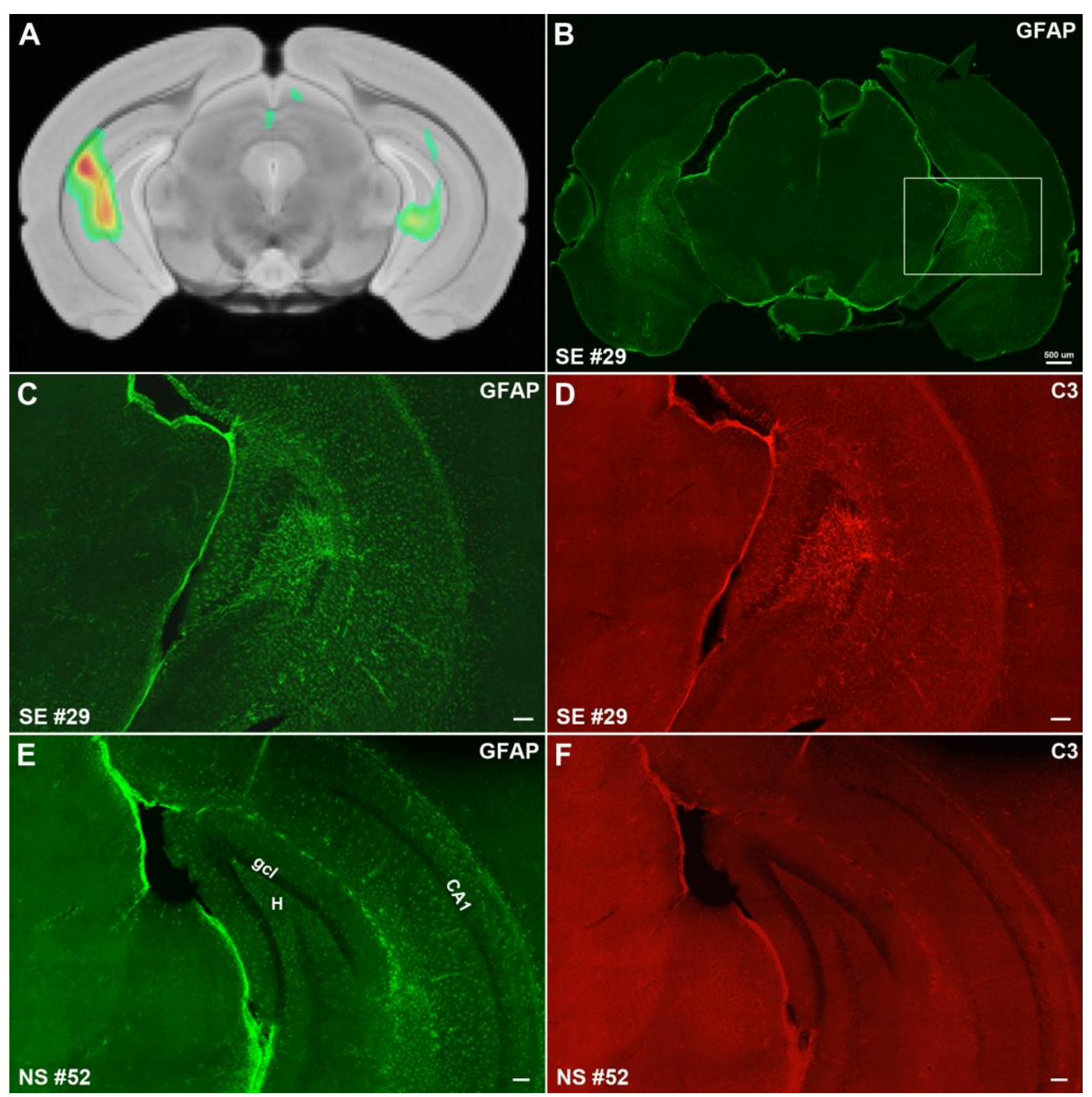

Fig. 9 


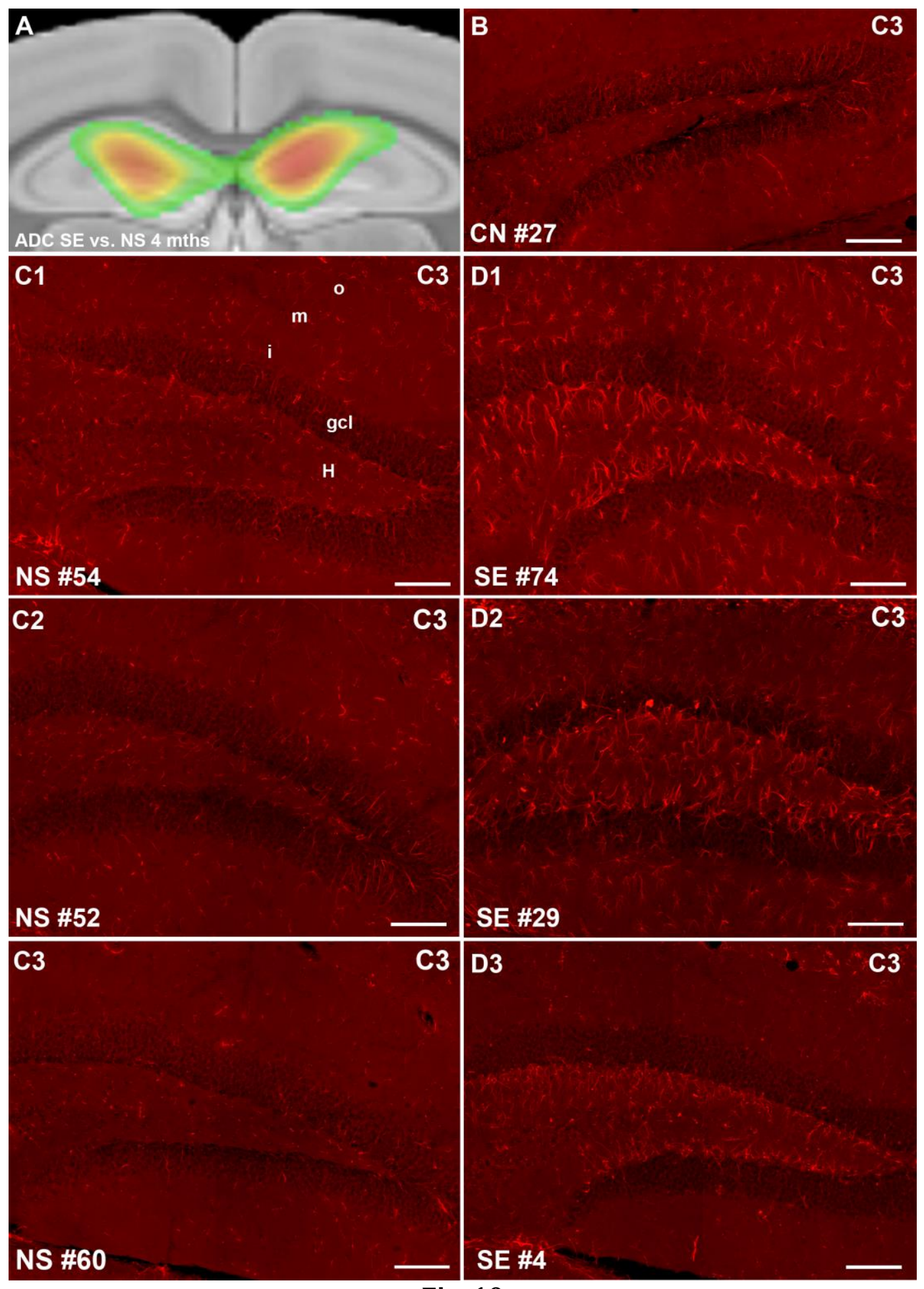

Fig. 10 
Page $\mathbf{4 1}$ of $\mathbf{4 2}$

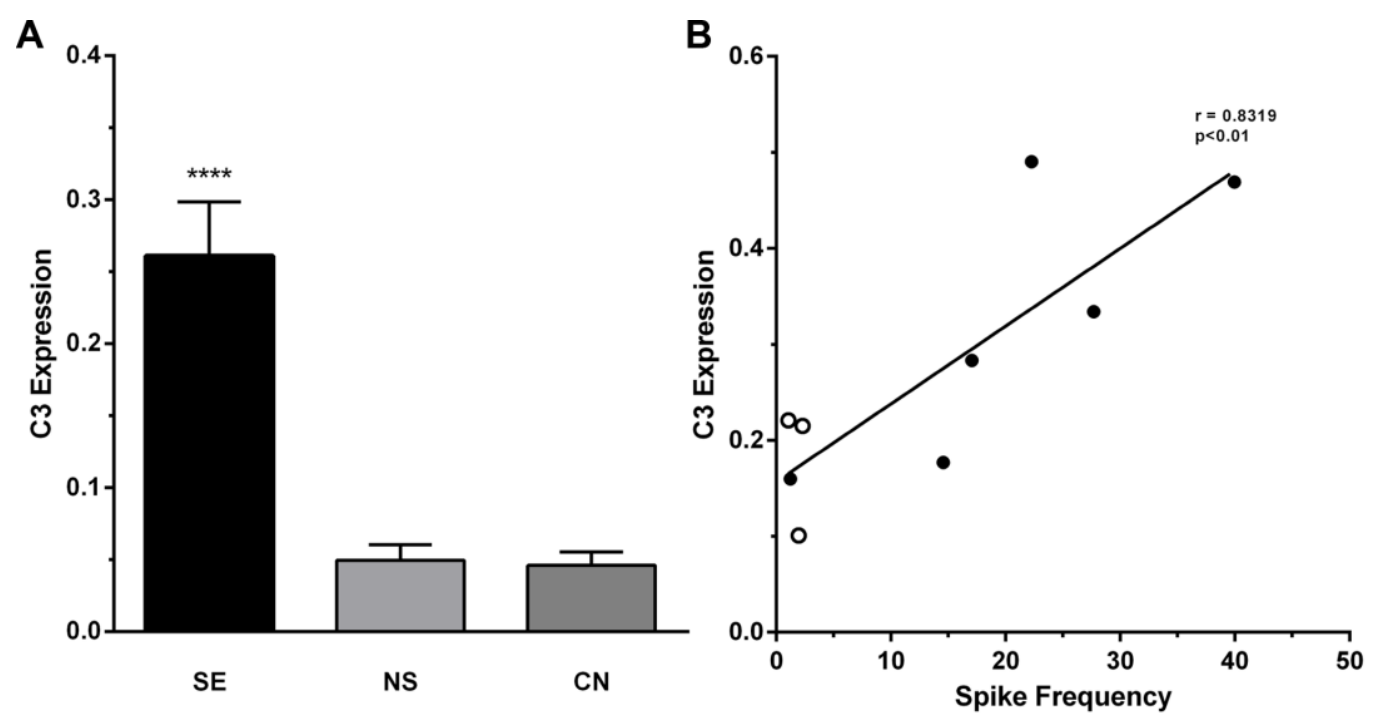

Fig. 11 
Page $\mathbf{4 2}$ of $\mathbf{4 2}$ 